\title{
AMPLE VECTOR BUNDLES ON COMPACT COMPLEX SPACES*
}

\author{
by Yozo Matsushima and Wilhelm Stoll
}

Consider an irreducible, compact space $X$ of pure dimension $m$. The field $\Re(X)$ of meromorphic functions on $X$ has a finite transcendence degree $\operatorname{tr}(X)$ over $\mathbf{C}$ with $0 \leqq \operatorname{tr}(X) \leqq m$. The space $X$ is called a Moišezon space if $\operatorname{tr}(X)=m$.

A holomorphic vector bundle $E$ over $X$ is said to be ample if finitely many global holomorphic sections $s_{0}, \cdots, s_{n}$ of $E$ over $X$ exist such that $s_{0}(x), \cdots, s_{n}(x)$ generate the vector space $E_{x}$ over $\mathbf{C}$ for each $x \in X$. Let $q$ be the fiber-dimension of $E$. The refined $v$ th Chern class $\hat{c}_{v}(E)$ is defined for $y=1, \cdots, q$. Take $\kappa=\left(\kappa_{1}, \cdots, \kappa_{q}\right)$ where each $\kappa_{v}$ is a non-negative integer. Define

$$
\sigma(\kappa)=\sum_{\nu=1}^{q} \nu \kappa_{\nu} .
$$

The refined Chern class of type $\kappa$ is defined by

$$
\hat{c}_{\kappa}(E)=\prod_{\nu=1}^{q} \hat{c}_{\nu}(E)^{\kappa_{\nu}}
$$

If $\sigma(\kappa)=m$, the Chern number

$$
C_{\kappa}(E)=\int_{X} \hat{c}_{\kappa}(E)
$$

of type $\kappa$ is defined. A refined Chern class is said to be non-negative, if it can be represented by a non-negative form.

The following results will be obtained:

I. If $E$ is ample, then $\hat{c}_{v}(E)$ and $\hat{c}_{\kappa}(E)$ are non-negative; especially, the Chern numbers of $E$ are non-negative (Proposition 4.2).

This result is due to Bott and Chern [5], section 5, but a less computa-

* This research was partially supported by the National Science Foundation under Grants NSF GP 29662 and NSF GP 20139. 
tional proof based on fiber integration will be provided here. The method may be of independent interest.

II. If $E$ is ample and if $\hat{c}_{\kappa}(E) \neq 0$, then $\operatorname{tr}(X) \geqq \sigma(\kappa)$. Especially, if a Chern number $C_{k}(E)$ of an ample bundle does not vanish, then $X$ is a Moišezon space (Theorem 5.5).

III. Main Theorem. If a connected, compact Kaehler manifold $X$ admits an ample holomorphic vector bundle $E$ with at least one nonvanishing Chern number $C_{\kappa}(E) \neq 0$, then $X$ is projective algebraic.

IV. Let $X$ be a connected, compact complex manifold of dimension $m$ with Euler characteristic $\chi(X)$. Let $V(X)$ be the vector space of holomorphic differential forms of bidegree $(1,0)$. Then the dimension $q(X)$ of $V(X)$ over $\mathbf{C}$ is finite and is called the irregularity of $X$. Let $T(x)$ be the holomorphic tangent bundle and $T^{*}(X)$ the holomorphic cotangent bundle. The Chern numbers of $T(X)$ are called the Chern numbers of $X$ and denoted by $C_{k}(X)$. If $T^{*}(X)$ is ample and $q(X)=m+1$, then $C_{\kappa}(X)=\chi(X)$ for all Chern numbers of $X$. If, in addition, $X$ is Kaehlerian and $\chi(X) \neq 0$, then $X$ is projective algebraic (Proposition 6.6).

V. Let $A$ be an abelian variety of dimension $m+1>2$. Then a connected, compact, projective algebraic, complex manifold $X$ of pure dimension $m$ with irregularity $q(X)=m+1$ and characteristic $\chi(X) \neq 0$ exists such that the following conditions are satisfied:

a) The holomorphic cotangent bundle of $X$ is ample.

b) The Albanese variety $A(X)$ of $X$ is a covering group of $A$; i.e., a finite subgroup $D$ of $A(X)$ exists such that

$$
A=A(X) / D \text {. }
$$

(Theorem 6.7).

\section{§1. Differential forms on complex spaces}

Recently, differential forms have been successfully used on complex spaces. Since this concept is new, a short outline shall be given here. See Bloom-Herrera [2], Cowen [6], Herrera [9], and King [10] for references.

Let $X$ be a complex space. ${ }^{1}$ Let $\Sigma(X)$ be the set of non-simple points of $X$. The set $X^{0}=X-\Sigma(X)$ of simple points is a manifold which is open and dense in $X$. A biholomorphic map $\alpha: U_{\alpha} \rightarrow U_{\alpha}^{\prime}$ of an open subset $U_{\alpha} \neq \varnothing$ of $X$ onto an analytic subset $U_{\alpha}^{\prime}$ of an open subset $G_{\alpha}$ of a complex vector space $V_{\alpha}$ is called a patch (at $a$ if $a \in U_{\alpha}$ ). Let $j_{\alpha}: U_{\alpha}^{\prime} \rightarrow G_{\alpha}$ be the inclusion map. The embedding dimension of $X$ at $a$ is defined by

$$
e_{a}=\min \left\{\operatorname{dim} V_{\alpha} \mid \alpha \text { patch at } a\right\} .
$$


If $\alpha$ and $\beta$ are patches at $a$ with $e_{a}=\operatorname{dim} V_{a}$, an open neighborhood $N^{\prime}$ of $\alpha(a)$ in $G_{\alpha}$ and a smooth, injective, holomorphic map $\sigma: N^{\prime} \rightarrow U_{\beta}^{\prime}$ exists such that $\sigma \circ j_{\alpha} \circ \alpha=j_{\beta} \circ \beta$ on $\left(j_{\alpha} \circ \alpha\right)^{-1}\left(N^{\prime}\right)$. If also $e_{a}=$ $\operatorname{dim} V_{\beta}$, then $\sigma: N^{\prime} \rightarrow \sigma\left(N^{\prime}\right)$ is biholomorphic and $\sigma\left(N^{\prime}\right)$ is open in $G_{\beta}$.

Let $\psi$ be a differential form of class $C^{k}$ on $X^{0}$. Then $\psi$ is called a differential form of class $C^{k}$ on $X$ if and only if for every $a \in X$ a patch $\alpha$ at $a$ and a form $\hat{\psi}$ of class $C^{k}$ on $G_{\alpha}$ exists such that $\left(j_{\alpha} \circ \alpha\right)^{*}(\hat{\psi})=\psi$ on $U_{\alpha}^{0}$. If $\psi$ is a form of class $C^{k}$ on $X$ and if $\alpha$ is a patch at $a$, an open neighborhood $N$ of $a$ in $U_{\alpha}$ and an open neighborhood $N^{\prime}$ of $\alpha(a)$ in $G_{\alpha}$ and a form $\hat{\psi}$ of class $C^{k}$ on $N^{\prime}$ exist such that $j_{\alpha}(\alpha(N)) \subseteq N^{\prime}$ and $\left(j_{\alpha} \circ \alpha\right)^{*}(\hat{\psi})=\psi$ on $N^{0}$. Here, class $C^{k}$ stands for any reasonable interpretation, as $k=0$ : "continuous," $1 \leqq k \leqq \infty$ : " $k$-times differentiable," $k=\rho$ : "real analytic," $k=\omega$ : "holomorphic." If $\psi$ has degree $m$, respectively bidegree $(p, q)$, then $\hat{\psi}$ can be chosen to have degree $m$, respectively bidegree $(p, q)$. This definition is consistent with the definition of continuous functions on $X$ and the definitions of classes of functions on complex manifolds.

For every open subset $U \neq \varnothing$ of $X$, let $A_{k}^{p, q}(U)$ be the vector space of forms of class $C^{k}$ and bidegree $(p, q)$ on $U$. If $V \neq \varnothing$ is open in $U$, the restriction map $r_{V}^{U}: A_{k}^{p, q}(U) \rightarrow A_{k}^{p, q}(V)$ is defined. The collection $A_{k}^{p, q}=$ $\left\{A_{k}^{p, q}(U), r_{V}^{U}\right\}$ is a canonical presheaf of vector spaces. Then

$$
A_{k}^{m}=\bigoplus_{p+q=m} A_{k}^{p, q} \text { and } A_{k}=\bigoplus_{m=0}^{\infty} A_{k}^{m}
$$

are the presheaves of forms of class $C^{k}$ and degree $m$, respectively of forms of class $C^{k}$ on $X$. Here $A_{k}$ carries the structure of an exterior graded, respectively bigraded, algebra over the presheaf $A_{k}^{0}$ of functions of class $C^{k}$, since the exterior product of forms of class $C^{k}$ has class $C^{k}$.

If $k \geqq 1$ and if $\psi$ is a form of class $C^{k}$ on $X$, then $d \psi, \partial \psi, \partial \psi$ and $d^{\perp} \psi$ are defined on $X^{0}$ and are forms of class $C^{k-1}$ on $X$. Here $d=\partial+\delta$ and $d^{\perp}=i(\partial-\partial \overline{)}$ with the usual properties, except Poincaré's Lemma.

The presheaf $A_{k}$ induces a sheaf $\mathfrak{A}_{k}$ on $X$. Each form $\psi \in A_{k}(X)$ defines a section in $\mathfrak{A}_{k}$. Herrera and King define this sheaf $\mathfrak{A}_{k}$ first and then define a form as a section in $\mathfrak{X}_{k}$. The effect is the same. Each form $\psi$ of class $C^{k}$ has a sheaf value $\psi_{x} \in\left(\mathfrak{U}_{k}\right)_{x}$ for each $x \in X$, but a function value $\psi(x)$ in the appropriate exterior power of the cotangent bundle exists only if $x \in X^{0}$.

Let $f: X \rightarrow Y$ be a holomorphic map between complex spaces. Let $\psi$ be a form of class $C^{k}$ on $Y$. Then one and only one form $f^{*}(\psi)$ of class $C^{k}$ on $X$ exists satisfying the condition: 
(P) Let $\alpha$ and $\beta$ be patches on $X$ and $Y$ respectively. Suppose that a holomorphic map $\tilde{f}: G_{\alpha} \rightarrow G_{\beta}$ exists such that

$$
\tilde{f} \circ j_{\alpha} \circ \alpha=j_{\beta} \circ \beta \circ f \text {. }
$$

Suppose that a form $\hat{\psi}$ on $G_{\beta}$ exists such that $\left(j_{\beta} \circ \beta\right)^{*}(\hat{\psi})=\psi$. Then

$$
f^{*}(\psi)=\left(j_{\alpha} \circ \alpha\right)^{*}\left(\tilde{f}^{*}(\psi)\right) \quad \text { on } U_{\alpha}^{0} .
$$

(For every point $a \in X$, patches $\alpha$ at $a$ and $\beta$ at $f(a)$ exist such that the extensions $\tilde{f}$ and $\hat{\psi}$ exist as required in (P).)

Because $f(X) \subseteq \Sigma(Y)$ may occur, the existence of $f^{*}$ is not trivial. The existence is known. Tung [20] will contain an alternative proof. The pull back $f^{*}$ has the same categorial properties as in the category of manifolds. It preserves degree and bidegree and commutes with $d, d^{\perp}, \partial, \bar{\partial}$ and the exterior product. If $f: X \rightarrow Y$ and $g: Y \rightarrow Z$ are holomorphic, then $(g \circ f)^{*}=f^{*} \circ g^{*}$.

Let $\psi$ be a differential form of bidegree $(p, p)$ on $X$. Then $\psi$ is said to be non-negative, i.e., $\psi \geqq 0$, (respectively positive, i.e., $\psi>0$ ) on $X$ if and only if the following condition is satisfied:

"Let $M$ be a pure $p$-dimensional analytic subset of an open subset $G$ of $X$ with $M^{0}=M$. Let $j: M \rightarrow X$ be the inclusion map. Then $j^{*}(\psi) \geqq 0$ (respectively $j^{*}(\psi)>0$ ) on $M$."

(Observe that $j^{*}(\psi)$ has degree $2 p$ on the $p$-dimensional complex manifold $M$. Hence $j^{*}(\psi) \geqq 0$ resp. $j^{*}(\psi)>0$ are well-defined.)

The form $\psi$ of bidegree $(p, p)$ is non-negative on $X$ if and only if for every holomorphic map $f: G \rightarrow X$ of an open subset $G$ of $\mathbf{C}^{p}$, the form $f^{*}(\psi)$ is non-negative on $G$. If $\psi$ is non-negative on $X$, and if $f: Y \rightarrow X$ is holomorphic, then $f^{*}(\psi)$ is non-negative on $Y$.

Let $\psi$ and $\chi$ be non-negative forms on $X$. If $\chi$ has bidegree $(1,1)$, then $\psi \wedge \chi$ is non-negative. It is unknown if this remains true if $\chi$ has bidegree $(q, q)$ with $q>1$ (Lelong conjecture). (Added in proof: Harvey disproved it.)

Let $X$ and $Y$ be complex manifolds of pure dimensions $m$ and $n$ respectively with $m-n=q \geqq 0$. Let $f: X \rightarrow Y$ be a regular ${ }^{2}$ holomorphic map. Then an operator $f_{*}$, called integration over the fibers, is defined. See [17] and [19] for the properties of this operator. If $\psi$ is a form of bidegree $(r, s)$ on $X$ with $r \geqq q$ and $s \geqq q$, and if $f \mid \operatorname{supp} \psi$ is proper, then the fiber integral $f_{*} \psi$ exists and is a form of bidegree $(r-q, s-q)$ on $Y$.

If $\psi$ has class $C^{k}$, so does $f_{*} \psi$. Unfortunately, $f_{*}(\psi \wedge \chi) \neq f_{*}(\psi) \wedge f_{*}(\chi)$, as already degree comparison shows. Nevertheless, the fiber integral of an exterior product can be expressed as the exterior product of fiber integrals. 
Since this seems to be unknown, and since this will be helpful later, it will be proven here.

Let $X, Y, N$ be complex manifolds of pure dimensions $k, m$, and $n$ respectively with $k-n=p \geqq 0$ and $m-n=q \geqq 0$. Let $f: X \rightarrow N$ and $g: Y \rightarrow N$ be holomorphic maps. A holomorphic map

$$
f \times g: X \times Y \rightarrow N \times N
$$

is defined by $(f \times g)(x, y)=(f(x), g(y))$. The diagonal

$$
\Delta_{N}=\{(z, z) \mid z \in N\}
$$

is analytic in $N \times N$ with $\Sigma\left(\Delta_{N}\right)=\varnothing$. A biholomorphic map $\delta_{N}: \Delta_{N} \rightarrow N$ is defined by $\delta_{N}(z, z)=z$. The inclusion map $j_{N}: \Delta_{N} \rightarrow N \times N$ is holomorphic and smooth. The set

$$
Z=X \underset{f, g}{X} Y=(f \times g)^{-1}\left(\Delta_{N}\right)=\{(x, y) \mid f(x)=g(y)\}
$$

is anaytic in $X \times Y$. The inclusion map $j: Z \rightarrow X \times Y$ is holomorphic. Because $(f \times g)(Z) \subseteq \Delta_{N}$, a holomorphic map

$$
f \wedge g=\delta_{N} \circ(f \times g) \circ j: Z \rightarrow X
$$

is defined. Then $\pi_{1}: Z \rightarrow X$ and $\pi_{2}: Z \rightarrow Y$ are holomorphic such that the diagram

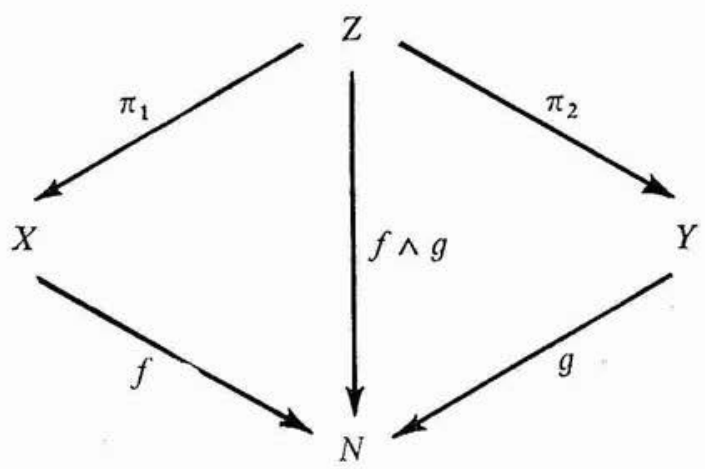

commutes. If $z \in N$, if $x \in X$, and if $y \in Y$, then

$$
\begin{array}{ll}
(f \wedge g)^{-1}(z) & =f^{-1}(z) \times g^{-1}(z) \\
\pi_{1}^{-1}(x) & =\{x\} \times g^{-1}(f(x)) \\
\pi_{2}^{-2}(y) & =f^{-1}(g(y)) \times\{y\} .
\end{array}
$$


Lemma 1.1. If $f$ and $g$ are regular, then $Z$ is a smooth, closed, complex submanifold of $X \times Y$ and the maps $f \wedge g, \pi_{1}$ and $\pi_{2}$ are regular. $Z$ has pure dimension $n+p+q$.

Proof. Take $(a, b) \in Z$. Define $c=(f \wedge g)(a, b)=f(z)=g(b)$. Then open subsets $U_{\alpha}, U_{\beta}, U, U_{\alpha}^{\prime}, U_{\beta}^{\prime}$ of $X, Y, N, \mathbf{C}^{p}, \mathbf{C}^{q}$ respectively and biholomorphic maps $\alpha: U_{\alpha} \rightarrow U_{\alpha}^{\prime} \times U$ and $\beta: U_{\beta} \rightarrow U_{\beta}^{\prime} \times U$ exist with $\rho_{1} \circ \alpha=f$ and $\rho_{2} \circ \beta=g$, where $\rho_{1}: U_{\alpha}^{\prime} \times U \rightarrow U$ and $\rho_{2}: U_{\beta}^{\prime} \times U \rightarrow U$ are the projections and where $a \in U_{\alpha}$ and $b \in U_{\beta}$ and $c \in U$. Then

$$
\left.Z^{\prime}=\{x, z, y, z) \mid x \in U_{\alpha}^{\prime}, z \in U_{\beta}^{\prime}, y \in U\right\}
$$

is a smooth, closed, complex submanifold of $U_{\alpha}{ }^{\prime} \times U \times U_{\beta}^{\prime} \times U$. The map

$$
\alpha \times \beta: U_{\alpha} \times U_{\beta} \rightarrow U_{\alpha}^{\prime} \times U \times U_{\beta}^{\prime} \times U
$$

is biholomorphic with $(\alpha \times \beta)\left(Z \cap\left(U_{\alpha} \times U_{\beta}\right)\right)=Z^{\prime}$. A biholomorphic map $\varepsilon: Z^{\prime} \rightarrow U_{\alpha}^{\prime} \times U_{\beta}^{\prime} \times U$ is defined by $\varepsilon(x, z, y, z)=(x, y, z)$. The map

$$
\gamma=\varepsilon \circ(\alpha \times \beta): Z \cap\left(U_{\alpha} \times U_{\beta}\right) \rightarrow U_{\alpha}^{\prime} \times U_{\beta}^{\prime} \times U
$$

is biholomorphic. Therefore, the analytic subset $Z$ of $X \times Y$ is a closed, smooth, complex submanifold of $X \times Y$. Let $\rho_{3}, \rho_{4}, \rho_{5}$ be the projections of $U_{\alpha}^{\prime} \times U_{\beta}^{\prime} \times U$ onto $U, U_{\alpha}^{\prime} \times U, U_{\beta}^{\prime} \times U$ respectively. Then $\rho_{3} \circ \gamma=f \wedge g$ and $\rho_{4} \circ \gamma=\alpha \circ \pi_{1}$ and $\rho_{5} \circ \gamma=\beta \circ \pi_{2}$ on $Z \cap\left(U_{\alpha} \times U_{\beta}\right)$. Therefore, the maps $f \wedge g, \pi_{1}$ and $\pi_{2}$ are regular; Q.E.D.

Theorem 1.2. Let $X, Y$ and $N$ be complex manifolds of pure dimension $k, m$, and $n$ respectively with $k-n=p>0$ and $m-n=q>0$. Let $f: X \rightarrow N$ and $g: Y \rightarrow N$ be regular, holomorphic maps. Define diagram (1.1). Let $\psi$ and $\chi$ be continuous forms of degree $r$ on $X$ and of degrees on Y respectively with $2 k \geqq r \geqq 2 p$ and $2 m \geqq s \geqq 2 q$. Suppose that $f \mid \operatorname{supp} \psi$ and $g \mid \operatorname{supp} \chi$ are proper. Then

$$
(f \wedge g)_{*}\left(\pi_{1}^{*}(\psi) \wedge \pi_{2}^{*}(\chi)\right)=f_{*}(\psi) \wedge g_{*}(\chi) .
$$

Here $f \wedge g \mid \operatorname{supp} \pi_{1}^{*}(\psi) \wedge \pi_{2}^{*}(\chi)$ is proper.

Proof. The results of [17] Appendix II will be used frequently. At first, observe that

$$
\begin{aligned}
& \operatorname{deg}\left(\pi_{1}^{*} \psi \wedge \pi_{2}^{*} \chi\right)=s+r \\
& \operatorname{deg}\left(f_{*} \psi \wedge g_{*} \chi\right)=s+r-2 p-2 q \\
& \operatorname{dim} Z=n+p+q, \quad \operatorname{dim} Y=n .
\end{aligned}
$$


Hence if $2 n+2 p+2 q<s+r$, then $\pi_{1}^{*}(\psi) \wedge \pi_{2}^{*}(\chi)=0$ and $f_{*}(\psi) \wedge g_{*}(\chi)=0$. Therefore, the theorem is true in this case.

Now assume $2 n+2 p+2 q \geqq s+r$. Consider diagram (1.1). Take $a \in X$. Define $b=f(z)$. Let $j_{b}: g^{-1}(b) \rightarrow Y$ and $\kappa: \pi_{1}^{-1}(a) \rightarrow Z$ be the inclusion map. The projection

$$
u_{a}: \pi_{1}^{-1}(a)=\{a\} \times g^{-1}(b) \rightarrow g^{-1}(b)
$$

is biholomorphic with $\pi_{2} \circ \kappa=j_{b} \circ u_{a}$. By [17] Theorem A II 4.15 $\pi_{1 *} \pi_{2}^{*} \chi$ exists with $\pi_{1 *} \pi_{2}^{*} \chi=f^{*} g_{*} \chi$. Now, [17] Lemma A II 4.6 implies

$$
\begin{aligned}
& \pi_{1 *}\left(\pi_{1}^{*}(\psi) \wedge \pi_{2}^{*}(\chi)\right)=\psi \wedge \pi_{1 *}\left(\pi_{2}^{*}(\chi)\right)=\psi \wedge f^{*}\left(g_{*}(\chi)\right) \\
& f_{*}\left(\psi \wedge f^{*}\left(g_{*}(\chi)\right)\right)=f_{*}(\psi) \wedge g_{*}(\chi) .
\end{aligned}
$$

Because

$$
S=\operatorname{supp}\left(\pi_{1}^{*}(\psi) \wedge \pi_{2}^{*}(\chi)\right) \subseteq Z \cap(\operatorname{supp} \psi \times \operatorname{supp} \chi)
$$

the map $f \wedge g\left|S=\delta_{N} \circ(f \times g)\right| S$ is proper. Hence $\pi_{1}^{*}(\psi) \wedge \pi_{2}^{*}(\chi)$ is tiber integrable over the fibers of $f \wedge g$.

Again, [17] Theorem A II 4.15 implies

$$
\begin{aligned}
(f \wedge g)_{*}\left(\pi_{1}^{*}(\psi)\right. & \left.\wedge \pi_{2}^{*}(\chi)\right) \\
& =f_{*}\left(\pi_{1 *}\left(\pi_{1}^{*}(\psi) \cap \pi_{2}^{*}(\chi)\right)\right) \\
& =f_{*}\left(\psi \wedge f^{*} g_{*}(\chi)\right) \\
& =f_{*}(\psi) \wedge g_{*}(\chi) ; \quad \text { Q.E.D. }
\end{aligned}
$$

This result also holds in the category of oriented differentiable manifolds, only great care has to be taken about signs and orientations. Let $X, Y$, and $N$ be oriented, real manifolds of class $C^{\infty}$ with pure, real dimensions $k$, $m$, and $n$ respectively. Assume $k-n=p>0$ and $m-n=q>0$. Let $f: X \rightarrow N$ and $g: Y \rightarrow N$ be regular maps of class $C^{\infty}$. Then $Z$ is a closed, oriented, smooth submanifold of $X \times Y$ such that $\gamma$ is an orientation preserving diffeomorphism if $\alpha$ and $\beta$ are. Let $\psi$ and $\chi$ be forms of degree $r$ on $X$ and of degree $s$ on $Y$ respectively. Assume $p \leqq r \leqq k$ and $q \leqq s \leqq m$. Suppose that $f \mid \operatorname{supp} \psi$ and $g \mid \operatorname{supp} \chi$ are proper. Then

$$
(f \wedge g)_{*}\left(\pi_{1}^{*}(\psi) \wedge \pi_{2}^{*}(\chi)\right)=(-1)^{(p-r) q} f_{*}(\psi) \wedge g_{*}(\chi) .
$$

Corollary 1.3. Let $N$ be a complex manifold of pure dimension $n$. For each $\mu=0,1, \cdots, m$, let $X_{\mu}$ be a complex manifold of dimension $k_{\mu}$ with $k_{\mu}-n=p_{\mu}>0$ and let $f_{\mu}: X_{\mu} \rightarrow N$ be a regular, holomorphic 
map. Let $\psi$ be a continuous, non-negative form of bidegree $\left(r_{0}, r_{0}\right)$ on $X_{0}$ with $p_{0} \leqq r_{0} \leqq k_{0}$ such that $f_{0} \mid \operatorname{supp} \psi$ is proper. For each $\mu=1, \cdots, m$, let $\chi_{\mu}$ be a continuous, non-negative form of bidegree $(1,1)$ on $X_{\mu}$ such that $f_{\mu} \mid \operatorname{supp} \chi_{\mu}$ is proper. Take $r_{\mu} \in \mathbf{Z}$ with $p_{\mu} \leqq r_{\mu} \leqq k_{\mu}$. Then

$$
f_{0 *}(\psi) \wedge f_{1 *}\left(\chi_{1}^{r_{1}}\right) \wedge \cdots \wedge f_{m *}\left(\chi_{m}^{r_{m}}\right) \geqq 0
$$

is non-negative on $N$.

Proof. By induction the following statement will be proven for $\mu=0,1, \cdots m$.

$\left(S_{\mu}\right)$. A complex manifold $Y_{\mu}$ of pure dimension $t_{\mu}$, a regular holomorphic map $g_{\mu}: Y_{\mu} \rightarrow N$ with $q_{\mu}=t_{\mu}-n \geqq 0$ and a non-negative, continuous form $\phi_{\mu}$ of bidegree $\left(s_{\mu}, s_{\mu}\right)$ with $s_{\mu} \geqq q_{\mu}$ exist such that $g_{\mu} \mid \operatorname{supp} \phi_{\mu}$ is proper and such that

$$
0 \leqq g_{\mu *}\left(\phi_{\mu}\right)=f_{0 *}(\psi) \wedge f_{1 *}\left(\chi_{1}^{1}\right) \wedge \cdots \wedge f_{\mu}^{*}\left(\chi_{\mu}^{r_{\mu}}\right) .
$$

$\left(S_{0}\right)$ is correct by [17] Lemma II 6.8, with $Y_{0}=X_{0}, g_{0}=f_{0}, t_{0}=k_{0}$, $q_{0}=p_{0}, \phi_{0}=\psi, s_{0}=r_{0}$.

Assume that $\left(S_{\mu}\right)$ is correct, then $\left(S_{\mu+1}\right)$ will be proven if $\mu+1 \leqq m$. According to Lemma 1.1,

$$
Y_{\mu+1}=Y_{\mu} \underset{g_{u}, f_{\mu+1}}{\times} X_{\mu+1}
$$

is a complex manifold of pure dimension $t_{\mu+1}=t_{\mu}+p_{\mu}$. Let $\pi_{1}: Y_{\mu+1} \rightarrow Y_{\mu}$ and $\pi_{2}: Y_{\mu+1} \rightarrow X_{\mu+1}$ be the projections. The map $g_{\mu+1}=g_{\mu} \wedge f_{\mu+1}$ : $Y_{\mu+1} \rightarrow N$ is holomorphic and regular. The form

$$
\phi_{\mu+1}=\pi_{1}^{*}\left(\phi_{\mu}\right) \wedge \pi_{2}^{*}\left(\chi_{\mu+1}^{r_{\mu+1}}\right)=\pi_{1}^{*}\left(\phi_{\mu}\right) \wedge \pi_{2}^{*}\left(\chi_{\mu+1}\right)^{r_{\mu+1}}
$$

is continuous, non-negative and has bidegree $\left(s_{\mu+1}, s_{\mu+1}\right)$ with

$$
s_{\mu+1}=s_{\mu}+r_{\mu} \geqq q_{\mu}+p_{\mu}=t_{\mu}-n+p_{\mu}=t_{\mu+1}-n=q_{\mu+1} .
$$

According to Theorem 1.2, $g_{\mu+1} \mid \operatorname{supp} \phi_{\mu+1}$ is proper and

$$
\begin{aligned}
\left(g_{\mu+1}\right)_{*}\left(\phi_{\mu+1}\right) & =\left(g_{\mu} \wedge f_{\mu+1}\right)_{*}\left(\pi_{1}^{*}\left(\phi_{\mu}\right) \wedge \pi_{2}^{*}\left(\chi_{\mu+1}^{r_{\mu+1}}\right)\right) \\
& =g_{\mu *}\left(\phi_{\mu}\right) \wedge\left(f_{\mu+1}\right)_{*}\left(\chi_{\mu+1}^{r_{\mu}}\right) \\
& =f_{0 *}(\psi) \wedge f_{1 *}\left(\chi_{1}^{1}\right) \wedge \cdots \wedge f_{\mu *}\left(\chi_{\mu}^{r_{\mu}}\right) \wedge\left(f_{\mu+1}\right)_{*}\left(\chi_{\mu+1}^{r_{\mu+1}}\right) .
\end{aligned}
$$

According to [17] Lemma A II $6.8,\left(g_{\mu+1}\right)_{*}\left(\phi_{\mu+1}\right) \geqq 0$. Hence the induction is completed. Statement $S_{m}$ is proved and implies the Corollary; Q.E.D. 


\section{\$2. Holomorphic vector bundles}

Let $E$ be a holomorphic vector bundle of fiber dimension $q$ over the complex space $X$. Then $s=\left(s_{1}, \cdots, s_{q}\right)$ is called a holomorphic frame over the open set $U$ of $X$, if and only if each $s_{\mu}: U \rightarrow E$ is a holomorphic section of $E$ over $U$ and if $s(x)=\left(s_{1}(x), \cdots, s_{q}(x)\right)$ is a basis for the vector space $E_{x}$ over $\mathbf{C}$ for each $x \in U$.

A hermitian metric along the fibers of $E$ is a function

$$
\text { (| ): } E \oplus E \rightarrow \mathbf{C}
$$

of class $C^{\infty}$ on the direct sum $E \oplus E$ such that the restriction

$$
(\mid)_{x}: E_{x} \times E_{x} \rightarrow \mathrm{C}
$$

to the fiber over $x$ is a positive definite hermitian form for each $x \in M$. A holomorphic vector bundle together with a hermitian metric along its fibers is called a hermitian vector bundle. Each holomorphic vector bundle admits a hermitian metric along its fibers.

Let $A$ be a hermitian vector bundle. Let $s=\left(s_{1}, \cdots, s_{q}\right)$ be a holomorphic frame over the open subset $U$ of $X$. The function $g_{\mu \nu}=\left(s_{\mu} \mid s_{v}\right)$ has class $C^{\infty}$ on $U$. Hence

$$
\begin{aligned}
H_{s} & =\left(\begin{array}{c}
g_{11}, \cdots, g_{1 q} \\
\vdots \\
g_{q 1}, \cdots, g_{q q}
\end{array}\right) \\
\eta_{s} & =\left(\partial H_{s}\right) H_{s}^{-1} \\
\Omega_{s} & =d \eta_{s}-\eta_{s} \wedge \eta_{s} \\
c(E, s) & =\sum_{v=0}^{q} c_{\nu}(E, s)=\operatorname{det}\left(I+\frac{i}{2 \pi} \Omega_{s}\right)
\end{aligned}
$$

are well defined on $U$ in the sense of section 1 . Here $I$ is the unit matrix and the form $c_{v}(E, s)$ has bidegree $(v, v)$ and class $C^{\infty}$ on $U$. These forms $c_{v}(E, s)$ and $c(E, s)$ do not depend on the choices of $s$ and $U$. Because the open sets $U$ carrying a holomorphic frame of $E$ over $U$ make up an open covering of $X$, global forms $c_{v}(E)$ of bidegree $(v, v)$ and class $C^{\infty}$ are uniquely defined on $X$ such that $c_{v}(E) \mid U=c_{v}(E, s)$ for each frame $s$. The form $c_{v}(E)$ is called the $v$ th Chern form of the hermitian vector bundle $E$. The direct sum $c(E)=\sum_{v=0}^{q} c_{v}(E)$ is called the total Chern form of the hermitian vector bundle $E$. Here $d c_{v}(E)=0$ for each $v$. If $v>q$, define $c_{v}(E)=0$. The Chern forms depend on the choice of the hermitian metric $(\mid)$ along the fibers of $E$. If $(\mid)^{\prime}$ is another choice, then a form $\rho_{\nu}$ of bidegree $(v-1, v-1)$ and of class $C^{\infty}$ exists on $X$ such that 


$$
c_{v}(E)-c_{v}^{\prime}(E)=d d^{\perp} \rho_{v}
$$

on $X$. These properties are proved in Bott and Chern [5] for manifolds; the same proofs work on complex spaces, see Cowen $[6]$.

Take $\kappa=\left(\kappa_{1}, \cdots, \kappa_{q}\right)$ with $0 \leqq \kappa_{\nu} \in \mathbf{Z}$ for $v=1, \cdots, q$. Define:

Then

$$
\sigma(\kappa)=\sum_{v=1}^{q} v \kappa_{v}
$$

$$
c_{\kappa}(E)=c_{1}(E)^{\kappa_{1}} \wedge \cdots \wedge c_{q}(E)^{\kappa_{q}}
$$

(where $c_{v}(E)^{0}=1$ ) is called a Chern form of type $\kappa$. Obviously, $c_{\kappa}(E)$ has bidegree $(\sigma(\kappa), \sigma(\kappa))$ and class $C^{\infty}$ on $X$ such that

$$
d c_{\kappa}(E)=0 .
$$

If another hermitian metric $(\mid)^{\prime}$ along the fibers of $E$ is chosen, a form $\rho_{\kappa}$ of bidegree $(\sigma(\kappa)-1, \sigma(\kappa)-1)$ and of class $C^{\infty}$ exists on $X$ such that

$$
c_{\kappa}(E)-c_{\kappa}^{\prime}(E)=d d^{\perp} \rho_{\kappa}
$$

provided $\sigma(\kappa) \geqq 1$.

Let $A^{p, q}$ be the complex vector space of forms of bidegree $(p, q)$ and of class $C^{\infty}$ on $X$. Then

$$
d=d_{p}: A^{p, p} \rightarrow A^{p+1, p} \oplus A^{p, p+1} .
$$

Define the refined de Rham groups by

$$
\begin{array}{ll}
\hat{H}^{p}(X)=\operatorname{ker} d_{p} / d d^{\perp} A^{p-1, p-1} & \text { if } p>0, \\
\hat{H}^{0}(X)=\operatorname{ker} d_{0} & \text { if } p=0 .
\end{array}
$$

Let $\rho: \operatorname{ker} d_{p} \rightarrow H^{p}(X)$ be the residual map. Then

$$
\hat{c}_{v}(E)=\rho\left(c_{v}(E)\right) \in \hat{H}^{v}(X)
$$

is called the $v$ th refined Chern class of $E$ and

$$
\hat{c}_{\kappa}(E)=\hat{c}_{1}(E)^{\kappa_{1}} \wedge \cdots \wedge \hat{c}_{q}(E)^{\kappa_{q}}=\rho\left(c_{\kappa}(E)\right) \in \hat{H}^{\sigma(\kappa)}(X)
$$

is called the refined Chern class of type $\kappa$. By (2.1) and (2.3) these refined Chern classes do not depend on the choice of the hermitian metric along the fibers of $E$. They are invariants of the holomorphic vector bundle $E$, well defined for each holomorphic vector bundle.

Consider the case where $X$ is compact and has pure dimension $m$. Take $\kappa=\left(\kappa_{1}, \cdots, \kappa_{q}\right)$ with $0 \leqq \kappa_{\mu} \in \mathbf{Z}$ for $\mu=1, \cdots, q$ such that $\sigma(\kappa)=m$. According to Lelong [11], the so called Chern number of type $\kappa$ 


$$
C_{\kappa}(E)=\int_{X} c_{\kappa}(E)
$$

exists and is independent of the choice of the hermitian metric along the fibers of $E$ (Stokes's Theorem). They are invariants of the holomorphic vector bundle $E$, well defined for each holomorphic vector bundle on the compact complex space $X$. According to Stokes's Theorem (Lelong $[11]), C_{\kappa}(E) \neq 0$ implies $\hat{c}_{\kappa}(E) \neq 0$.

If $X$ is a compact complex manifold of pure dimension $m$ and if $E=T(X)$ is the holomorphic tangent bundle, then

$$
C_{\kappa}(X)=C_{\kappa}(T(X))
$$

is called the Chern number of type $\kappa$ of $X$.

Again, let $E$ be a holomorphic vector bundle of fiber dimension $q$ over the complex space $X$. Let $E^{*}$ be the dual bundle. For each hermitian metric along the fibers of $E$, one and only one hermitian metric along the fibers of $E^{*}$, called the dual metric, exists such that $E_{x}^{*}$ carries the dual metric to the metric on $E_{x}$. Hence the hermitian vector bundle $E$ defines canonically the dual hermitian vector bundle $E^{*}$. An easy computation shows

$$
\begin{aligned}
& c_{v}\left(E^{*}\right)=(-1)^{\frac{y}{c}} c_{v}(E) \quad(y=0,1, \cdots, q) \\
& c_{\kappa}\left(E^{*}\right)=(-1)^{\sigma(\kappa)} c_{\kappa}(E)
\end{aligned}
$$

if $\kappa=\left(\kappa_{1}, \cdots, \kappa_{q}\right)$ with $0 \leqq \kappa_{v} \in Z$. If $X$ is compact and has pure dimension $m=\sigma(\kappa)$, then

$$
C_{\kappa}\left(E^{*}\right)=(-1)^{m} C_{\kappa}(E) .
$$

Let $\tilde{X}$ and $X$ be complex spaces. Let $\pi: E \rightarrow X$ be a holomorphic vector bundle over $X$. Let $f: \tilde{X} \rightarrow X$ be a holomorphic map. Then a holomorphic vector bundle $\tilde{\pi}: \widetilde{E} \rightarrow \tilde{X}$ and a holomorphic map $\tilde{f}: \widetilde{E} \rightarrow E$ exist such that the diagram

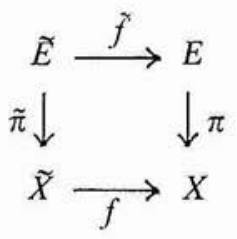

commutes and such that the restriction $\tilde{f}_{x}=\tilde{f}: \widetilde{E}_{x} \rightarrow E_{f(x)}$ is a linear isomorphism for each $x \in \tilde{X}$. This pull back is uniquely determined by these conditions up to a holomorphic isomorphism of the diagram (2.4). One possible choice within the isomorphic models is 


$$
\tilde{E}=\tilde{X} \underset{f, \pi}{\times} E=\{(x, e) \in \tilde{X} \times E \mid f(x)=\pi(e)\}
$$

where $\tilde{\pi}: \widetilde{E} \rightarrow \tilde{X}$ and $\tilde{f}: \tilde{E} \rightarrow E$ are the projections.

Take a hermitian metric (|) along the fibers of $E$. One and only one hermitian metric $(\mid)$ along the fibers of $\tilde{E}$, called the pull back, is defined by $\left(e_{1}, e_{2}\right)^{\sim}=\left(\tilde{f}\left(e_{1}\right), \tilde{f}\left(e_{2}\right)\right)$ if $\left(e_{1}, e_{2}\right) \in \tilde{E} \oplus \tilde{E}$.

Let $s=\left(s_{1}, \cdots, s_{q}\right)$ be a holomorphic frame of $E$ over the open subset $U$ of $X$ with $\tilde{U}=f^{-1}(U) \neq \varnothing$. Define $\tilde{s}_{\mu}: \tilde{U} \rightarrow \tilde{E}$ by $\tilde{s}_{\mu}(x)=\tilde{f}_{x}^{-1}\left(s_{\mu}(f(x))\right)$ for $x \in \tilde{U}$. Then $\tilde{s}=\left(\tilde{s}_{1}, \cdots, \tilde{s}_{q}\right)$ is a holomorphic frame of $\tilde{E}$ over $\tilde{U}$ with $s_{\mu} \circ f=\tilde{f} \circ \tilde{s}_{\mu}$ for $\mu=1, \cdots, q$. Then

$$
\begin{aligned}
\tilde{g}_{u v}=\left(\tilde{s}_{\mu} \mid \tilde{s}_{v}\right)^{\sim} & =\left(\tilde{f} \circ \tilde{s}_{\mu} \mid \tilde{f} \circ \tilde{s}_{v}\right) \\
& =\left(s_{u} \circ f \mid s_{v} \circ f\right)=g_{\mu v} \circ f
\end{aligned}
$$

implies

$$
\begin{aligned}
H_{\tilde{s}} & =H_{s} \circ f & \eta_{\tilde{s}} & =f^{*}\left(\eta_{s}\right) \\
\Omega_{\tilde{s}} & =f^{*} \Omega_{s} & c_{v}(\tilde{E}, \tilde{s}) & =f^{*}\left(c_{\nu}(E, s)\right) .
\end{aligned}
$$

Hence

$$
\begin{aligned}
& c_{v}(E)=f^{*}\left(c_{v}(E)\right) \quad \text { for } v=1, \cdots, q, \\
& c_{\kappa}(E)=f^{*}\left(c_{\kappa}(E)\right),
\end{aligned}
$$

if $\kappa=\left(\kappa_{1}, \cdots, \kappa_{q}\right)$ with $0 \leqq \kappa_{\mu} \in \mathbf{Z}$ for $\mu=1, \cdots, q$.

Consider a short exact sequence

$$
0 \rightarrow D \stackrel{\leftrightarrow}{\rightarrow} E \stackrel{\rho}{\rightarrow} F \rightarrow 0
$$

of hermitian vector bundles over $X$. Then a form $\rho=\rho_{0}+\cdots+\rho_{q-1}$ of class $C^{\infty}$ with bidegree $\rho=(v, v)$ for $v=0, \cdots, q-1$ exists on $X$ such that

$$
\begin{aligned}
c(E) & =c(D) \wedge c(F)+d d^{\perp} \rho, \quad \text { i.e., } \\
c_{\lambda}(E) & =\sum_{\mu+\nu=\lambda} c_{\mu}(D) \wedge c_{\nu}(E)+d d \rho_{\lambda-1}
\end{aligned}
$$

for $\lambda=0, \cdots, q$. (For manifolds, see Bott and Chern [5], for complex spaces, see Cowen [6])

Assume that (2.7) is only a short exact sequence of holomorphic vector bundles over $X$. Assume a hermitian metric $(\mid)$ along the fibers of $E$ is given. It restricts to a hermitian metric along the fibers of $D$. Differentiably, the exact sequence (2.7) splits into an exact sequence 


$$
0 \rightarrow F \stackrel{j}{\rightarrow} E \stackrel{\sigma}{\rightarrow} D \rightarrow 0
$$

such that $j$ and $\sigma$ are differentiable bundle homomorphisms with $\sigma \circ \mathbf{t}$, $\rho \circ j, \imath \circ \sigma+j \circ \rho$ being identity maps and such that $(j(f) \mid \imath(d))_{x}=0$ for all $f \in F_{x}, d \in D_{x}$ and $x \in X$. Then $F$ can be considered as a subbundle of $E$. The hermitian metric (|) along the fibers of $E$ restricts to a hermitian metric along the fibers of $F$. Hence a hermitian metric along the fibers of $E$ induces hermitian metrics along the fibers of $D$ and $F$ by this process, which shall be called orthonormal metrization.

Let $V$ be a complex vector space of finite dimension. A positive definite hermitian form $(\mid)$ on $V$ is called a hermitian metric on $V$. A complex vector space together with a hermitian metric is called a hermitian vector space. If $E=X \times V$ is the trivial bundle, a hermitian metric on $V$ defines a hermitian metric along the fibers of $X \times V$ by

$$
((x, v) \mid(x, w))=(v \mid w) \text { if } x \in X, v \in V, w \in V .
$$

The norm of $z \in V$ is defined by $|z|=\sqrt{(z \mid z)}$.

\section{§3. Grassmann manifolds}

Let $V$ be a complex vector space of dimension $n+1$ with $n>0$. On $V-\{0\}$, an equivalence relation is defined by " $a \sim b$ if and only if $a \wedge b=0$." The equivalence class of $\mathfrak{a}$ is denoted by $\mathbf{P}(\mathfrak{a})$. If $A \subseteq V$, define

$$
\mathbf{P}(A)=\{\mathbf{P}(a) \mid 0 \neq a \in V\} .
$$

Note $\mathbf{P}(A)=\mathbf{P}(A-\{0\})$. The quotient space $\mathbf{P}(V)$ is a connected, compact, complex manifold of dimension $n$, called the projective space associated to $V$. The residual map

$$
\mathbf{P}: V-\{0\} \rightarrow \mathbf{P}(V)
$$

is holomorphic, 1-fibering, regular, and surjective. If $W$ is a linear subspace of dimension $p+1$ of $V$ with $p \geqq 0$, then $\mathbf{P}(W)$ is a $p$-dimensional, smooth, compact, complex submanifold of $\mathbf{P}(V)$, called a projective plane of dimension $p$. Let $\mathfrak{L}_{p}(V)$ be the set of all $p$-dimensional linear subspaces of $V$. Let $\mathfrak{G}_{p}(V)$ be the set of all $p$-dimensional projective planes in $\mathbf{P}(V)$.

The $p$-fold exterior product of $V$ is denoted by $V^{[p]}=V \wedge \cdots \wedge V$. For $0 \leqq p<n$, the Grassmann cone

$$
\tilde{G}_{p}(V)=\left\{\mathfrak{v}_{0} \wedge \cdots \wedge \mathfrak{p}_{p} \mid \mathfrak{v}_{\mu} \in V\right\}
$$

is an analytic subset of $V^{[p+1]}$. The Grassmann manifold $G_{p}(V)=$ 
$\mathbf{P}\left(\tilde{G}_{p}(V)\right)$ is a connected, compact, smooth, complex submanifold of $\mathbf{P}\left(V^{[p+1]}\right)$ with dimension

and with degree

$$
k(p)=(n-p)(p+1)
$$

$$
d(p, n)=\frac{p ! \cdots 1 !}{(n-p) ! \cdots n !} k(p) !
$$

If $a \in G_{p}(V)$, then $0 \neq a \in \widetilde{G}_{p}(V)$ with $\mathbf{P}(a)=a$ exists. Then

$$
\begin{aligned}
& \tilde{E}(a)=\{3 \in V \mid z \wedge a=0\} \in \mathfrak{Q}_{p+1}(V) \\
& E(a)=P(\tilde{E}(a)) \in \mathfrak{G}_{p}(V)
\end{aligned}
$$

are well-defined. If $a=a_{0} \wedge \cdots \wedge a_{p}$, then

The maps

$$
\widetilde{E}(a)=\mathrm{Ca}_{0}+\cdots+\mathrm{Ca}_{p} \text {. }
$$

$$
\tilde{E}: G_{p}(V) \rightarrow \mathbb{Q}_{p+1}(V) \text { and } E: G_{p}(V) \rightarrow \mathfrak{G}_{p}(V)
$$

are bijective. Note $G_{0}(V)=\mathbf{P}(V)$.

Let $\Gamma(V)$ be the set of all bases $a=\left(a_{0}, \cdots, a_{n}\right)$ of $V$. Define the matrix space

$$
\mathbf{C}_{p}^{n}=\left\{\left(\begin{array}{ccc}
z_{0, p+1}, & \cdots, & z_{0, n} \\
\vdots & & \vdots \\
z_{p, p+1} & \cdots & z_{p, n}
\end{array}\right) \mid z \in \mathbf{C}\right\} .
$$

For $\mathfrak{a}=\left(\mathfrak{a}_{0}, \cdots, \mathfrak{a}_{n}\right) \in \Gamma(V)$, define

$$
\begin{aligned}
& \tilde{Z}_{\mathfrak{a}}=\left\{\mathfrak{v} \in G_{p}(V) \mid \mathfrak{v} \wedge a_{p+1} \wedge \cdots \wedge a_{n} \neq 0\right\} \\
& Z_{\mathfrak{a}}=\mathbf{P}(\tilde{Z}) \subseteq G_{p}(V) .
\end{aligned}
$$

According to [15] Lemma 2.1, $Z_{\mathfrak{a}}$ is open in $G_{p}(V)$ and a biholomorphic map

$$
\zeta_{\mathfrak{a}}: \mathbf{C}_{p}^{n} \rightarrow Z_{\mathrm{a}}
$$

is defined as follows: "Define holomorphic vector functions $\mathfrak{v}_{\mu}: \mathbf{C}_{p}^{n} \rightarrow V-\{0\}$ for $\mu=0, \cdots, p$ by

Then

$$
\mathfrak{b}_{\mu}(z)=a_{\mu}+\sum_{\nu=p+1}^{n} z_{\mu \nu} a_{v} \text { for } z \in \mathbf{C}_{p}^{n} .
$$

$$
\tilde{\zeta}_{\boldsymbol{a}}=\mathfrak{p}_{0} \wedge \cdots \wedge \mathfrak{p}_{p}: \mathbf{C}_{p}^{n} \rightarrow \tilde{Z}_{\mathfrak{a}}-\{0\}
$$

is holomorphic and 


$$
\zeta_{a}=\mathbf{P} \circ \tilde{\zeta}_{\mathfrak{a}}: \mathbf{C}_{p}^{n} \rightarrow Z_{\alpha}
$$

is biholomorphic." Define $\xi_{a}=\zeta_{a}^{-1}: Z_{a} \rightarrow \mathrm{C}_{p}^{n}$. Then $\left\{\xi_{a}\right\}_{a \in \mathrm{r}(V)}$ is a complex atlas of $G_{p}(V)$, because $\left\{Z_{a}\right\}_{a \in \Gamma(V)}$ is an open covering of $G_{p}(V)$. The coordinate functions $\xi_{\mu \nu}=\xi_{\mu \nu}^{\mathfrak{a}}$ of the matrix functions $\xi_{a}$ are holomorphic on $Z_{\mathfrak{a}}$. Holomorphic vectors $\mathrm{w}_{\mu}: Z_{\mathfrak{a}} \rightarrow V$ are defined by

$$
\mathfrak{w}_{\mu}=\mathfrak{w}_{\mu}^{\mathfrak{a}}=\mathfrak{p}_{\mu} \circ \xi_{\mathfrak{a}}=\mathfrak{a}_{\mu}+\sum_{\nu=p+1}^{n} \xi_{\mu v} \mathfrak{a}_{v} .
$$

Then

$$
\mathbf{P}\left(\mathfrak{w}_{0}(x) \wedge \cdots \wedge \mathfrak{w}_{p}(x)\right)=\zeta_{\mathfrak{a}} \circ \xi_{\mathfrak{a}}(x)=x
$$

for all $x \in Z_{\mathfrak{a}}$. Hence

$$
\widetilde{E}(x)=\mathbf{C w}_{0}(x)+\cdots+\mathrm{Cw}_{p}(x) \text { if } x \in Z_{\mathbf{a}} .
$$

Define

$$
S_{p}(V)=\bigcup_{x \in G_{p}(V)}\{x\} \times \tilde{E}(x)=\left\{(x, \mathfrak{v}) \in G_{p}(V) \times V \mid \mathfrak{v} \in \tilde{E}(X)\right\} .
$$

Lemma 3.1. $S_{p}(V)$ is a holomorphic subbundle of the trivial bundle $G_{p}(V) \times V$.

Proof. Take any $a=\left(a_{0}, \cdots, a_{n}\right) \in \Gamma(V)$. Then

$$
\mathfrak{w}_{0}^{\mathfrak{a}} \wedge \cdots \wedge \mathfrak{w}_{p}^{\mathfrak{a}} \wedge \mathfrak{a}_{p+1} \wedge \cdots \wedge \mathfrak{a}_{n} \neq 0
$$

on $Z_{\mathfrak{a}}$. Hence a biholomorphic map

$$
\phi_{\mathrm{a}}: Z_{\mathrm{a}} \times \mathbf{C}^{n+1} \rightarrow Z_{\mathrm{a}} \times V
$$

is defined by

$$
\phi_{\mathrm{a}}\left(x, z_{0}, \cdots, z_{n}\right)=\left(x, \sum_{\mu=1}^{p} z_{\mu} \mathrm{w}_{\mu}(x)+\sum_{\mu=p+1}^{n} z_{\mu} \mathfrak{a}_{\mu}\right) .
$$

Obviously, $\phi_{\mathfrak{a}}$ is a vector bundle isomorphism. Moreover,

$$
\phi_{\mathrm{a}}\left(Z_{\mathrm{a}} \times \mathbf{C}^{p+1}-\{0\}\right)=S_{p}(V) \cap\left(Z_{\mathrm{a}} \times V\right) .
$$

Because $\left\{Z_{\mathfrak{a}} \times V\right\}_{\mathfrak{a} \in \mathrm{r}(V)}$ is an open covering of $G_{p}(V) \times V$, the set $S_{p}(V)$ is a smooth complex submanifold of $G_{p}(V) \times V$.

A biholomorphic map $\psi_{\mathfrak{a}}: Z_{\mathfrak{a}} \times \mathbf{C}^{p+1} \rightarrow S_{p}(V) \cap\left(Z_{\mathfrak{a}} \times V\right)$ is defined by $\psi_{a}\left(x, z_{0}, \cdots, z_{p}\right)=\phi_{a}\left(x, z_{0}, \cdots, z_{p}, 0, \cdots, 0\right)$. Let $j: S_{p}(V) \rightarrow G_{p}(V) \times V$ be the inclusion. Define $j^{\prime}$ by $j^{\prime}(x, z)=(x, z, 0)$ for $(x, z) \in Z_{a} \times \mathbf{C}^{p+1}$ with $0 \in \mathbf{C}^{n-p}$. 


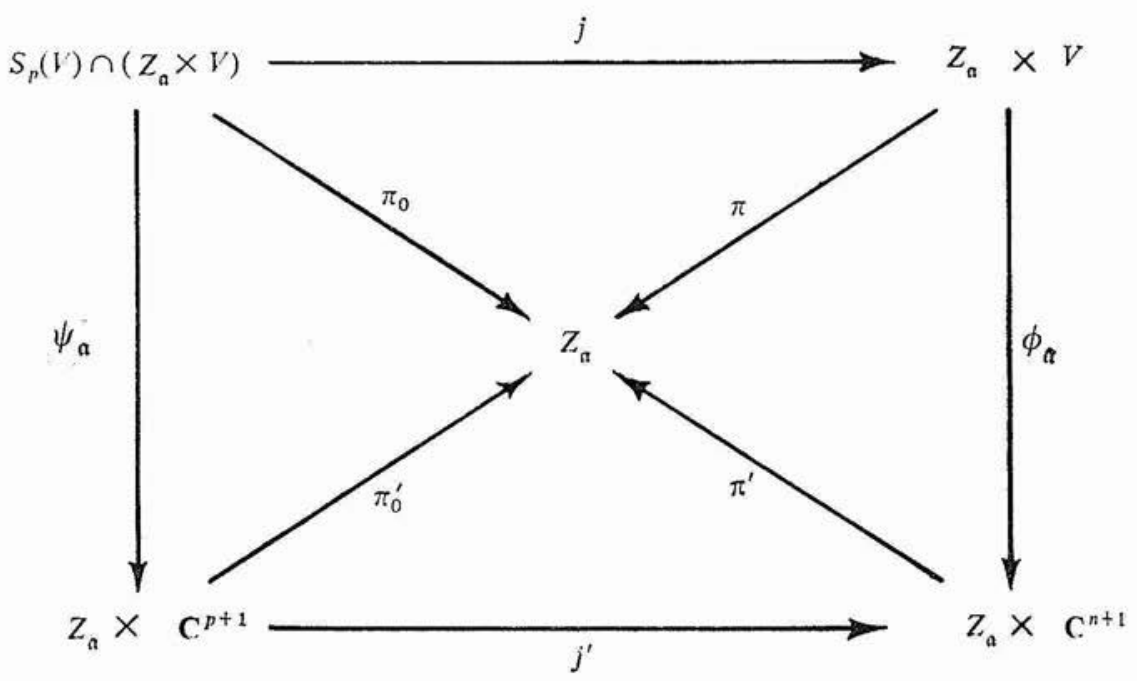

commutes, where $\pi, \pi_{0}, \pi_{0}^{\prime}, \pi^{\prime}$ are the projections. The maps $\psi_{\mathfrak{a}}, \phi_{a}, j$ and $j$ are linear on the fibers of the projections. Since $\left\{Z_{a}\right\}_{a \in \Gamma(V)}$ is an open covering of $G_{p}(V)$, these diagrams show that $S_{p}(V)$ is a holomorphic vector subbundle of $G_{p}(V) \times V$; Q.E.D.

Let $Q_{p}(V)$ be the quotient bundle. A short exact sequence

$$
0 \rightarrow S_{p}(V) \rightarrow G_{p}(V) \times V \rightarrow Q_{p}(V) \rightarrow 0
$$

of holomorphic vector bundles over $G_{p}(V)$ is constructed, called the classifying sequence.

According to [16], Lemma 1.1, the flag manifolds

$$
F_{j k}=\left\{(x, z) \in G_{j}(V) \times G_{k}(V) \mid E(x) \geqq E(z)\right\} \text { if } 0 \leqq k \leqq j \leqq n
$$

are smooth, connected, compact, complex submanifolds of $G_{j}(V) \times G_{k}(V)$ and the projections

$$
\pi: F_{j k} \rightarrow G_{k}(V) \quad \tau: F_{j k} \rightarrow G_{j}(V)
$$

are surjective, holomorphic, and regular. The map

$$
\text { Id } \times \mathbf{P}: G_{p}(V) \times(V-\{0\}) \rightarrow G_{p}(V) \times \mathbf{P}(V)
$$

restricts to

$$
\text { Id } \times \mathbf{P}: S_{p}(V)-\mathfrak{D} \rightarrow F_{p 0}
$$

where $\mathfrak{D}$ is the zero section of $S_{p}(V)$. Hence $F_{p 0}$ is the associated projective bundle $\mathbf{P}\left(S_{p}(V)\right)$ to $S_{p}(V)$. 
Take a hermitian metric on the vector space $V$. It defines a hermitian metric along the fibers of $G_{p}(V) \times V$. The process of orthonormal metrization defines hermitian metrics along the fibers of $S_{p}(V)$ and $Q_{p}(V)$. The Chern forms of these hermitian vector bundles will be considered.

The exterior product $V^{[p]}$ becomes a hermitian vector space with

$$
\left(a_{1} \wedge \cdots \wedge a_{p} \mid b_{1} \wedge \cdots \wedge b_{p}\right)=\left|\begin{array}{c}
\left(a_{1} \mid \mathfrak{b}_{1}\right), \cdots,\left(a_{1} \mid b_{p}\right) \\
\vdots \\
\left(a_{p} \mid b_{1}\right), \cdots,\left(a_{p} \mid b_{p}\right)
\end{array}\right| .
$$

One and only one positive form $\hat{1}_{p}$ of bidegree $(1,1)$ and class $C^{\infty}$ exists on $\mathbf{P}\left(V^{[p+1]}\right)$ such that

$$
\mathbf{P}^{*}\left(\hat{\omega}_{p}\right)(3)=\frac{1}{2 \pi} d^{\perp} d \log |3| \text { for } 0 \neq z \in V^{[p+1]}
$$

Let $j: G_{p}(V) \rightarrow \mathbf{P}\left(V^{[p+1]}\right)$ be the inclusion. Then $\omega_{p}=j^{*}\left(\Theta_{p}\right)$ is a positive form of bidegree $(1,1)$ and of class $C^{\infty}$ on $G_{p}(V)$. Obviously, $\omega_{0}=\omega_{0}$. The degree of $G_{p}(V)$ is given by ${ }^{3}$

$$
d(p, n)=\int_{G p(V)} \omega_{p}^{k}(p)
$$

Representation Theorem of Bott and Chern. For $0 \leqq q<p \leqq n$ consider the diagram

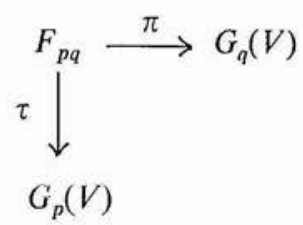

If $g>0$, then

$$
d(p-1, q-1) c_{\mu}\left(Q_{p}(V)\right)=\tau_{*} \pi^{*}\left(c_{\mu+p-q}\left(Q_{q}\right) \wedge \omega_{q}^{(p-q) q}\right)
$$

for $\mu=0, \cdots, n-p$. If $q=0$, then

$$
c_{\mu}\left(Q_{p}(V)\right)=\tau_{*} \pi^{*}\left(\omega_{0}^{p+\mu}\right)
$$

for $\mu=0, \cdots, n-p$.

Remark 1. For $\mu=n-p$, the theorem was stated first in Bott and Chern [5] (10.5); however, their statement is wrong for $q>0$ as degree comparison shows.

Remark 2. The complete theorem was announced in [19]. A proof will be given elsewhere. 
Remark 3. The form $\omega_{0}^{p+\mu}$ is positive. Hence $\pi^{*}\left(\omega_{0}^{p+\mu}\right) \geqq 0$. By [17] Lemma A II 6.8, $c_{\mu}\left(Q_{p}(V)\right)=\tau_{*} \pi^{*}\left(\omega_{0}^{p+\mu}\right) \geqq 0$. Hence the $\mu$ th Chern form of $Q_{p}(V)$ is non-negative for $\mu=0,1, \cdots, n-p$. Take $\kappa=\left(\kappa_{1}, \cdots, \kappa_{n-p}\right)$ with $0 \leqq \kappa_{\mu} \in \mathbf{Z}$. According to Corollary 1.3 , the Chern form $0 \leqq c_{\kappa}\left(Q_{p}(V)\right)$ $=c_{1}\left(Q_{p}(V)\right)^{\kappa_{1}} \wedge \cdots \wedge c_{n-p}\left(Q_{p}(V)\right)^{\kappa_{n-p}}$ of type $\kappa$ is non-negative.

Remark 4. Take another hermitian metric $(\mid)^{\prime}$ on $V$. Distinguish the derived forms by '. A positive function $g$ of class $C^{\infty}$ is defined on $\mathbf{P}(V)$ by $g(z)=|z|^{\prime} /|z|$ if $z \in \mathbf{P}^{-1}(z)$. Hence

$$
\omega_{0}^{\prime}=\omega_{0}+\frac{1}{2 \pi} d^{\perp} d \log g
$$

or

$$
\left(\omega_{0}^{\prime}\right)^{p+n}=\omega_{0}^{p+\mu}+d^{\perp} d \gamma_{n+p}
$$

where $\gamma_{\mu+p}$ is a form of bidegree $(p+\mu-1, p+\mu-1)$ and of class $C^{\infty}$. The Representation Theorem and [17] Lemma A II 6.7 imply

$$
c_{\mu}^{\prime}\left(Q_{p}(V)\right)=c_{\mu}\left(Q_{p}(V)\right)+d^{\perp} d \rho_{p+u}
$$

with $\rho_{p+u}=\tau_{*} \pi^{*}\left(\gamma_{p+u}\right)$. Also

$$
c_{\kappa}^{\prime}\left(Q_{p}(V)\right)=c_{\kappa}\left(Q_{p}(V)\right)+d^{\perp} d \rho_{\kappa},
$$

where $\rho_{\kappa}$ is a form of bidegree $(\sigma(\kappa)-1, \sigma(\kappa)-1)$ and of class $C^{\infty}$.

Remark 5. The forms $c_{\mu}\left(Q_{p}(V)\right)$ and $c_{\kappa}\left(Q_{p}(V)\right)$ are closed because,

$$
\begin{aligned}
d c_{\mu}\left(Q_{p}(V)\right) & =d \tau_{*} \pi^{*}\left(\omega_{0}^{p+u}\right)=\tau_{*} \pi^{*}\left(d \omega_{0}^{p+u}\right) \\
& =\tau_{*} \pi^{*}(0)=0 .
\end{aligned}
$$

The results of Remarks 3, 4, and 5 also follow from the complicated computations in Bott and Chern [5] Sections 3 and 5.

\$4. The classifying space for ample vector bundles

Let $E$ be a holomorphic vector bundle of fiber dimension $q$ on the complex space $X$. Then $E$ is said to be ample if and only if $E$ is the quotient bundle of a trivial bundle, i.e., a finite dimensional complex vector space $V$ and a surjective, holomorphic, vector bundle homomorphism

$$
\varepsilon: X \times V \rightarrow E
$$

exists. For each $\mathfrak{v} \in V$, a holomorphic section $\varepsilon_{y}$ in $E$ over $X$ is defined by $\varepsilon_{v}(x)=(x, \mathfrak{v})$. Let $\Gamma(X, E)$ be the complex vector space of global holomorphic sections $s: X \rightarrow E$. A linear map $\sigma: V \rightarrow \Gamma(X, E)$ is defined by 
$\sigma(\mathfrak{y})=\varepsilon_{\mathfrak{b}}$. The image $W=\sigma(V)$ is a finite dimensional linear subspace of $\Gamma(X, E)$. A surjective, holomorphic vector bundle homomorphism

$$
e: X \times W \rightarrow E
$$

is given by $e(x, s)=s(x)$ if $s \in W$, called the evaluation map. Obviously $\varepsilon=e \circ($ Id $\times \sigma)$. Therefore (4.2) gives an alternative definition for $E$ to be ample. Obviously, an equivalent condition is the requirement that finitely many sections $s_{0}, \cdots, s_{n}$ in $\Gamma(X, E)$ exist such that $s_{0}(x), \cdots, s_{n}(x)$ generate $E_{x}$ over $\mathbf{C}$ for each $x \in X$. Also, if $X$ is compact, $E$ is ample if and only if for every $x \in X$ at least one holomorphic section $s$ in $E$ over $X$ exists such that $s(x) \neq 0$.

Again, consider the situation (4.1) with $\operatorname{dim} V=n+1$. The kernel $S$ of $\varepsilon$ has fiber dimension $p+1$ with $p=n-q$. Then

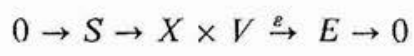

is a short exact sequence of holomorphic vector bundles over $X$ called an amplification of $E$ by $V$. To each such amplification, a so-called classifying map

$$
f: X \rightarrow G_{p}(V)
$$

is associated by

$$
E(f(x))=\mathbf{P}\left(S_{x}\right) \quad \text { if } x \in X .
$$

Observe, that $S_{x} \in \mathscr{Q}_{p+1}(V)$ and $\mathbf{P}\left(S_{x}\right) \in \mathfrak{F}_{p}(V)$. Since $E: G_{p}(V) \rightarrow \mathfrak{W}_{p}(V)$ is bijective, $f(x) \in G_{p}(V)$ is well defined.

Proposition 4.1. The classifying map $f$ is holomorphic. The amplification (4.3) is the pull back of the classifying sequence (3.1) under $f$; i.e.,

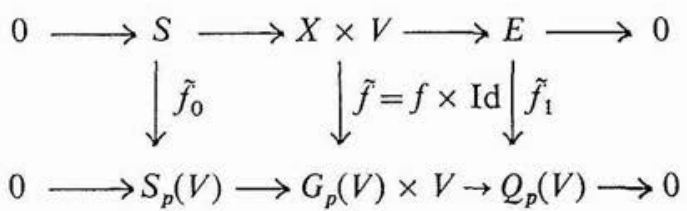

where $f_{0}, \tilde{f}, \tilde{f}_{1}$ are bundle homomorphisms over $f$, isomorphic in each fiber.

Proof. Take $a \in X$. A base $a=\left(a_{0}, \cdots, a_{n}\right)$ of $V$ exists such that $\varepsilon_{a_{p+1}}(a), \cdots, \varepsilon_{a_{n}}(a)$ is a basis for $E_{a}$. Abbreviate $s_{\mu}=\varepsilon_{\mathfrak{a}_{\mu}}$ for $\mu=0, \cdots, n$. An open neighborhood $U$ of $a$ exists such that $s_{p+1}(x), \cdots, s_{n}(x)$ is a base of $E_{x}$ for each $x \in U$. Holomorphic functions $b_{\mu \nu}$ exist on $U$ such that 


$$
s_{u}(x)=-\sum_{\nu=p+1}^{n} b_{\mu v}(x) s_{v}(x)
$$

for $\mu=0, \cdots, p$ if $x \in U$. Holomorphic vector functions

$$
\mathfrak{y}_{u}=\mathfrak{a}_{\mu}+\sum_{v=p+1}^{n} b_{\mu v}(x) \mathfrak{a}_{v}: U \rightarrow V
$$

are defined. If $x \in U$, then $\mathfrak{y}_{0}(x), \cdots, \mathfrak{y}_{p}(x)$ are linearly independent over $\mathbf{C}$. Hence a holomorphic map

$$
g: U \rightarrow G_{p}(V)
$$

is defined by

$$
g(x)=\mathbf{P}\left(\mathfrak{y}_{0}(x) \wedge \cdots \wedge \mathfrak{y}_{p}(x)\right) \text { for } x \in U .
$$

Moreover

$$
\varepsilon\left(x, \mathfrak{y}_{u}(x)\right)=s_{\mu}(x)+\sum_{v=p+1}^{n} b_{\mu v}(x) s_{v} x=0 .
$$

Hence $\mathfrak{y}_{0}(x), \cdots, \mathfrak{y}_{p}(x)$ is a base of $S_{x}$. Therefore

$$
S_{x}=\mathbf{C y}_{0}(x)+\cdots+\mathfrak{C y}_{p}(x)=E\left(\mathbf{P}\left(\mathfrak{y}_{0}(x) \wedge \cdots \wedge \mathfrak{y}_{p}(x)\right),\right.
$$

which implies

$$
E(f(x))=\mathbf{P}\left(S_{x}\right)=E\left(\mathbf{P}\left(\mathfrak{y}_{0}(x) \wedge \cdots \wedge \mathfrak{y}_{p}(x)\right)\right)=E(g(x))
$$

if $x \in U$. Because $E$ is bijective, $f \mid U=g: U \rightarrow G_{p}(V)$ is holomorphic. The map $f$ is holomorphic.

The matrix function

$$
b=\left(\begin{array}{c}
b_{0, p+1}, \cdots, b_{0, n} \\
\vdots \\
\vdots \\
b_{p, p+1}, \cdots, \\
b_{p, n}
\end{array}\right): U \rightarrow \mathbf{C}_{p}^{n}
$$

is holomorphic. Then $\mathfrak{y}_{\mu}=\mathfrak{p}_{\mu} \circ b$ and

$$
f \mid U=g=\mathbf{P}\left(\mathfrak{p}_{0} \circ b \wedge \cdots \wedge \mathfrak{p}_{p} \circ b\right)=\zeta_{a} \circ b: U \rightarrow Z_{a}
$$

where $Z_{a}$ is open in $G_{p}(V)$. Hence $\xi_{a} \circ f=b: U \rightarrow \mathbf{C}_{p}^{n}$ which implies

$$
\mathfrak{y}_{\mu}=\mathfrak{p}_{\mu} \circ \xi_{\alpha} \circ f=\mathfrak{w}_{\mu} \circ f: U \rightarrow V .
$$

If $x \in U$, then 


$$
\begin{aligned}
\tilde{f}\left(S_{x}\right) & =\tilde{f}\left(\{x\} \times\left(\mathbf{C y}_{0}(x)+\cdots+\mathbf{C y}_{p}(x)\right)\right. \\
& =\{f(x)\} \times\left(\mathbf{C y}_{0}(x)+\cdots+\mathbf{C y}_{p}(x)\right) \\
& =\{f(x)\} \times\left(\mathbf{C w}_{0}\left(f(x)+\cdots+\mathbf{C w}_{p}(f(x))\right)=S_{p}(V)_{f(x)} .\right.
\end{aligned}
$$

Because this is true for some neighborhood $U$ of an arbitrary point $a \in X$, the bundle homomorphism $\tilde{f}=f \times \mathrm{Id}: X \times V \rightarrow G_{p}(V) \times V$ restricts to $\tilde{f}_{0}: S \rightarrow S_{p}(V)$. Since the quotient bundle is uniquely defined up to isomorphisms, the diagram (4.6) is established; Q.E.D.

An immediate consequence is

Proposition 4.2. Let $E$ be an ample holomorphic vector bundle of fiber dimension q over the complex space $X$. Let (4.3) be an amplification of $E$ with $\operatorname{dim} V=n+1$ and $p=n-q>0$. Let $f: X \rightarrow G_{p}(V)$ be the associated classifying map. Take a hermitian metric on $V$. It defines a hermitian metric along the fibers of $X \times V$ and $G_{p}(V) \times V$. By orthonormal metrization, hermitian metrics along the fibers of $S, E, S_{p}(V)$ and $Q_{p}(V)$ are defined. With these metrics the following statements hold.

1. The $\mu$ th Chern form $c_{u}(E)$ of $E$ is non-negative and given by

$$
c_{\mu}(E)=F^{*}\left(c_{\mu}\left(Q_{p}(V)\right) \geqq 0 \text { with } d c_{\mu}(E)=0 .\right.
$$

2. If $\kappa=\left(\kappa_{1}, \cdots, \kappa_{q}\right)$ with $0 \leqq \kappa_{\mu} \in \mathbf{Z}$, then the Chern form $c_{\kappa}(E)$ of type $\kappa$ is non-negative and given by

$$
c_{\kappa}(E)=f^{*}\left(c_{\kappa}\left(Q_{p}(V)\right)\right) \geqq 0 \text { with } d c_{\kappa}(E)=0 .
$$

3. If $X$ is compact and pure m-dimensional, if $\kappa=\left(\kappa_{1}, \cdots, \kappa_{q}\right)$ with $0 \leqq \kappa_{\mu} \in \mathbf{Z}$ and $\sigma(\kappa)=m$, then the Chern number $C_{\kappa}(E)$ of type $\kappa$ is nonnegative.

4. If another hermitian metric $(\mid)^{\prime}$ on $V$ is given and if the associated forms are distinguished by ', then

$$
\begin{aligned}
& c_{\mu}^{\prime}(E)=c_{\mu}(E)+d d^{\perp} \gamma_{\mu} \\
& c_{\kappa}^{\prime}(E)=c_{\kappa}(E)+d d^{\perp} \gamma_{\kappa}
\end{aligned}
$$

where $\gamma_{\mu}$ and $\gamma_{k}$ are forms of class $C^{\infty}$ and bidegree $(\mu-1, \mu-1)$ and $(\sigma(\kappa)-1, \sigma(\kappa)-1)$ respectively. If $X$ is compact and pure dimensional the Chern numbers remain unchanged.

The proof follows immediately from Proposition 4.1, from (2.5) and (2.6) and from Remarks 3,4, and 5 in Section 3. The results of Proposition 4.2 follow also from the difficult computation of Bott and Chern [5]. Here, they are obtained from the classifying sequence, an idea already 
indicated in Bott and Chern [5], and which can be carried further, see [18] Section 14.

\section{$\S 5$. Moišezon spaces}

Let $\Omega(X)$ be the field of meromorphic functions on the irreducible complex space $X$. The transcendence degree of $\Re(X)$ over $\mathbf{C}$ is denoted by $\operatorname{tr}(X)$. If $X$ is compact, then $\operatorname{tr}(X) \leqq \operatorname{dim} X$. The case, $\operatorname{tr}(X)=\operatorname{dim} X$, has been extensively studied by Moišezon [13] and is not far from the algebraic case. Hence, a compact, irreducible complex space $X$ is called a Moišezon space if and only if $\operatorname{tr}(X)=\operatorname{dim} X$. If, in addition, $X=X^{0}$ is a manifold, then $X$ is called a Moišezon manifold. A complex space is said to be projective algebraic if and only if it is biholomorphically equivalent to an analytic subset of a projective complex space. According to a deep result of Moišezon [13], a Moišezon manifold is projective algebraic if and only if it is a Kaehler manifold.

Let $X$ and $Y$ be complex spaces. Suppose that $X$ is pure dimensional. Let $f: X \rightarrow Y$ be a holomorphic map. The rank of $f$ at $x \in X$ is defined by

$$
\operatorname{rank}_{x} f=\operatorname{dim}_{x} X-\operatorname{dim}_{x} f^{-1} f(x) .
$$

The global rank of $f$ is defined by

$$
\operatorname{rank} f=\sup \left\{\operatorname{rank}_{x} f \mid x \in X\right\} .
$$

Then $0 \leqq \operatorname{rank} f \leqq \min (\operatorname{dim} X, \operatorname{dim} Y)$. If $0 \leqq p \in \mathbf{Z}$, then

$$
E(p)=\left\{x \in X \mid \operatorname{rank}_{x} f \leqq p\right\}
$$

is analytic in $X$. If $X$ is irreducible. then

$$
D_{f}=\left\{x \in X \mid \operatorname{rank}_{x} f<\operatorname{rank} f\right\}
$$

is a thin analytic subset of $X$ (See [1] Section 1).

Lemma 5.1. Let $X$ and $Y$ be irreducible, compact, complex spaces of dimension $m$ and $n$ respectively. Let $f: X \rightarrow Y$ be a holomorphic map of global rank $n$. Suppose that $Y$ is a Moišezon space. Then $\operatorname{tr}(X) \geqq n$. Especially, if $m=n$ then $X$ is a Moišezon space.

Proof. Because $\operatorname{dim} Y=\operatorname{rank}_{f}$, the homomorphism $f^{*}: \mathfrak{R}(Y) \rightarrow \Omega(X)$ is injective. Hence $f^{*} \Re(Y)$ is a subfield of transcendence degree $n$ of $\Re(X)$. Therefore $\operatorname{tr}(X) \geqq n$. If $n=m$, then $n=m \geqq \operatorname{tr}(X) \geqq n$. Hence $X$ is a Moišezon space; Q.E.D. 
Lemma 5.2. An irreducible, projective algebraic, complex space is a Moišezon space.

Proof. Let $X$ be an irreducible, analytic subset of a complex projective space $\mathbf{P}(V)$ with $\operatorname{dim} X=m$. Here, $V$ is a complex vector space of dimension $n+1$. Then $n-m=p \geqq 0$. If $p=0$, then $X=\mathbf{P}(V)$ is a Moišezon space. Assume the statement is proved for $p-1 \geqq 0$. Take $X$ with $\operatorname{dim} X=m=n-p$. Take $a \in \mathbf{P}(V)-X$. Let $Z=\mathbf{P}(W) \in \mathfrak{5}_{n-1}(V)$ be a projective plane of dimension $n-1$ in $\mathbf{P}(V)$ with $a \in \mathbf{P}(V)-Z$. For each $x \in X$, one and only one projective plane $L_{x} \in \mathfrak{F}_{1}(V)$ of dimension 1 exists with $\{a, x\} \subseteq L_{x}$ because $a \neq x$. Since $a \notin Z$, one and only one point $\pi(x) \in L_{x} \cap Z$ exists. Because $a \notin L_{x} \cap X=\pi^{-1} \pi(x)$, the holomorphic map $\pi: X \rightarrow Z$ has rank $m$. Since $X$ is compact and irreducible, $Y=\pi(X)$ is an $m$-dimensional, irreducible analytic subset of $Z$ with $\operatorname{dim} Z-m=p-1$. By the induction assumption, $Y$ is a Moišezon space. The restriction $\pi: X \rightarrow Y$ has rank $m$. According to Lemma 5.1, $X$ is a Moišezon space; Q.E.D.

Lemma 5.3. Let $X$ be a compact, irreducible, complex space of dimension $m$. Let $Y$ be a projective algebraic complex space. Let $f: X \rightarrow Y$ be a holomorphic map of rank $p$. Then $\operatorname{tr}(X) \geqq p$. Especially, if $p=m$, then $X$ is a Moišezon space.

Proof. According to the Remmert proper mapping theorem, the image $N=f(X)$ is a compact, irreducible, analytic subset of $Y$ with $\operatorname{dim} N=p$. Hence $N$ is projective algebraic. By Lemma 5.2, $N$ is a Moišezon space. The restriction $f: X \rightarrow N$ has rank $p$. By Lemma $5.1, \operatorname{tr}(X) \geqq p$. If $p=m$, then $X$ is a Moišezon space; Q.E.D.

Lemma 5.4. Let $X$ and $Y$ be complex spaces. Let $f: X \rightarrow Y$ be a holomorphic map. Assume that $X$ is irreducible. Suppose $\psi$ is a continuous differential form of degree $2 p$ on $Y$ such that $f *(\psi) \neq \equiv$ on $X$. Then rank $f \geqq p$.

Proof. Define $n=\operatorname{rank} f$. Suppose that $n<p$. Take $a \in X-D_{f}$. Then $\operatorname{rank}_{x} f=n$ for all $x \in X-D_{f}$. According to Remmert [14], open neighborhoods $V$ of $a$ in $X-D_{f}$ and $W$ of $f(z)$ in $Y$ exist such that $Z=f(X)$ is a pure $n$-dimensional, analytic subset of $W$. (Also see [1], Proposition 1.21). Hence $f=j \circ f_{0}$, where $j: Z \rightarrow Y$ is the inclusion and where $f_{0}: V \rightarrow Z$ is the restriction. Then

$$
f^{*}(\psi) \mid V=f_{0}^{*}\left(j^{*}(\psi)\right)
$$


Now $\operatorname{deg} \psi=2 p>2 n$ and $n=\operatorname{dim} Z$ imply $j^{*}(\psi) \equiv 0$. Hence $f^{*}(\psi) \mid V \equiv 0$. Therefore $f^{*}(\psi) \equiv 0$ on $X-D_{f}$. Because $D_{f}$ is thin, $f^{*}(\psi) \equiv 0$, which is wrong. Therefore $n \geqq p$; Q.E.D.

Theorem 5.5. Let $X$ be a compact, irreducible complex space of dimension $m$. Let $E$ be an ample holomorphic vector bundle on $X$. Suppose that a refined Chern class $\hat{c}_{\kappa}(E) \neq 0$ exists with $\sigma(\kappa)=r>0$. Then $\operatorname{tr}(x) \geqq r$.

Proof. Take an hermitian vector space $V$ of dimension $n+1$ such that (4.3) is an amplification of $E$. Then $X \times V$ is a hermitian vector bundle. Introduce hermitian metrics along the fibers of $S$ and $E$ by orthonormal metrization. Let $g$ be the fiber dimension of $E$. Define $p=n-q$. Then $S$ has fiber dimension $p+1$. Let $f: X \rightarrow G_{p}(V)$ be the classifying map associated to the amplification (4.3). Then

$$
c_{\kappa}(E)=f *\left(c_{\kappa}\left(Q_{p}(V)\right)\right)
$$

by Proposition 4.2. Because $\hat{c}_{\kappa}(E) \neq 0$, also $c_{\kappa}(E) \neq 0$. According to Lemma 5.4, $\operatorname{rank} f \geqq r=\sigma(\kappa)$. Since $G_{p}(V)$ is projective algebraic, Lemma 5.3 implies $\operatorname{tr}(X) \geqq \operatorname{rank} f \geqq r$; Q.E.D.

Theorem 5.6. Let $X$ be a compact, irreducible, complex space. Let $E$ be an ample holomorphic vector bundle on $X$. Suppose that at least one Chern number $C_{k}(E) \neq 0$. Then $X$ is a Moišezon space.

Proof. Define $m=\operatorname{dim} X$. Then $\sigma(\kappa)=m$ and $c_{\kappa}(E) \neq 0$, since $C_{\kappa}(E) \neq 0$. Theorem 5.5 implies $\operatorname{tr}(X) \geqq m$. Therefore $\operatorname{tr}(X)=m$; Q.E.D.

Main Theorem. Let $X$ be a connected, compact Kaehler manifold. Let $E$ be an ample holomorphic vector bundle on $X$. Suppose that $E$ has at least one Chern number $C_{\kappa}(E) \neq 0$. Then $X$ is projective algebraic.

Proof. By Theorem 5.6, $X$ is a Moišezon space; hence $X$ is projective algebraic according to Moišezon [1.3]; Q.E.D.

§6. Manifolds with ample holomorphic tangent and cotangent bundles

Let $X$ be a complex manifold of pure dimension $m$. Let $T(X)$ be the holomorphic tangent bundle of $X$. Its sections are the holomorphic forms of bidegree $(1,0)$. The set of all biholomorphic maps $\sigma: X \rightarrow X$ is a group Aut $(X)$ called the automorphism group of $X$. The complex manifold $X$ is said to be homogeneous if and only if $\operatorname{Aut}(X)$ operates transitively on $X$. The following lemma is well-known. 
Lemma 6.1. Let $X$ be a connected, compact, complex manifold. Then $X$ is homogeneous if and only if the holomorphic tangent bundle $T(X)$ is ample.

The case of homogeneous compact complex manifolds is well studied. The following theorem is known. (See Goto [7], Borel and Remmert [4] and Grauert and Remmert [8].) It can be obtained easily from the amplification sequence:

Theorem 6.2. A connected, compact, homogeneous complex manifold $X$ with non-zero Euler characteristic $\chi(X) \neq 0$ is projective algebraic and each Chern number of $X$ is non-negative.

Proof. Because the Chern numbers of $X$ are the Chern numbers of the ample holomorphic tangent bundle $T(X)$, they are non-negative by Proposition 4.2. Let $V=\Gamma(X, T(X))$ be the vector space of all holomorphic vector fields on $X$. Then $n+1=\operatorname{dim} V<+\infty$. Define $m=\operatorname{dim} X$. Because $T(X)$ is ample, an amplification is defined by

$$
0 \rightarrow S \rightarrow X \times V \stackrel{e}{\rightarrow} T(X) \rightarrow 0
$$

where $e$ is the evaluation map. Because $\chi(X) \neq 0$, the map is not isomorphic. Hence $n+1>m$. Define $p=m-n \geqq 0$. The kernel $S$ has fiber dimension $p+1$. Let $f: X \rightarrow G_{p}(V)$ be the associated classifying map. Take a hermitian metric on $V$. It defines a hermitian metric along the fibers of $X \times V$ which induces hermitian metrics along the fibers of $S$ and $T(X)$ by orthonormal metrization. Then

$$
0 \neq \chi(X)=\int_{X} c_{m}(T(X))=\int_{X} f\left(c_{m}\left(Q_{p}(V)\right)\right) .
$$

Hence $f^{*}\left(c_{m}\left(Q_{p}(V)\right)\right) \stackrel{\neq}{\neq}$. Lemma 5.4 implies rank $f \geqq m$, hence rank $f=m$. Therefore, $Y=f(X)$ is a compact, irreducible, $m$-dimensional analytic subset of $G_{p}(V)$. It shall be shown that $Y$ is a smooth, homogeneous, complex submanifold of $G_{p}(V)$ and that $f: X \rightarrow Y$ is locally biholomorphic.

For this purpose, consider the operations of the group Aut $(X)$. Take $\sigma \in \operatorname{Aut}(X)$. Then $\sigma: X \rightarrow X$ is biholomorphic and induces a holomorphic vector bundle isomorphism $\sigma_{*}: T(X) \rightarrow T(X)$ over $\sigma$. It defines a linear isomorphism $\sigma_{*}: V \rightarrow V$ which extends to an isomorphism

$$
\sigma_{*}=\sigma \times \sigma_{*}: X \times V \rightarrow X \times V
$$

such that $\sigma_{*} \circ e=e \circ \sigma_{*}$. Hence $\sigma_{*}$ restricts to $\sigma_{*}: S \rightarrow S$. An automorphism of (6.1) is defined 


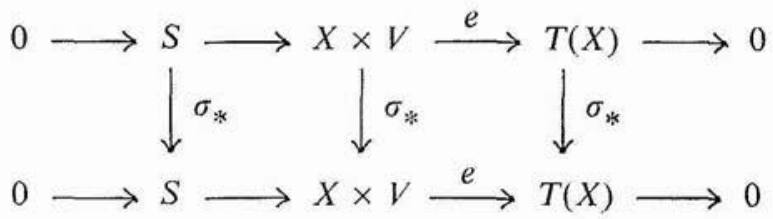

If $t \in \mathrm{N}$, the linear isomorphism $\sigma_{*}: V \rightarrow V$ extends $\sigma_{*}: V^{[t]} \rightarrow V^{[t]}$ such that $\sigma_{*}\left(v_{1} \wedge \cdots \wedge v_{t}\right)=\sigma_{*}\left(v_{1}\right) \wedge \cdots \wedge \sigma_{*}\left(v_{t}\right)$. This isomorphism defines a biholomorphic map

$$
\sigma_{*}: \mathbf{P}\left(V^{[t]}\right) \rightarrow \mathbf{P}\left(V^{[t]}\right)
$$

such that $\sigma_{*} \circ \mathbf{P}=\mathbf{P} \circ \sigma_{*}$, which restricts to

$$
\sigma_{*}: G_{t-1}(V) \rightarrow G_{t-1}(V) \text {. }
$$

If $a \in G_{t-1}(V)$, then $\sigma_{*}(\widetilde{E}(a))=\tilde{E}\left(\sigma_{*}(a)\right)$. Hence

$$
\sigma_{*}(E(a))=E\left(\sigma_{*}(a)\right) \text {. }
$$

Take $x \in X$. Then $f(x) \in G_{p}(V)$. Therefore

$$
\begin{aligned}
E\left(\sigma_{*}(f(x))\right) & =\sigma_{*}(E(f(x)))=\sigma_{*}\left(\mathbf{P}\left(S_{x}\right)\right)=\mathbf{P}\left(\sigma_{*}\left(S_{x}\right)\right) \\
& =\mathbf{P}\left(S_{\sigma(s)}\right)=E(f(\sigma(x)) .
\end{aligned}
$$

Consequently, $\sigma_{*} \circ f=f \circ \sigma$. Hence

$$
\sigma_{*}(Y)=\sigma_{*}(f(X))=f(\sigma(X))=f(X)=Y .
$$

Hence $\sigma_{*}: G_{p}(V) \rightarrow G_{p}(V)$ restricts to a biholomorphic map

$$
\sigma_{*}: Y \rightarrow Y \text {. }
$$

If $y_{\mu} \in Y$, then $x_{n} \in Y$ with $y_{n}=f\left(x_{n}\right)$ exist for $\mu=1,2$. Take $\sigma \in \operatorname{Aut}(X)$ with $\sigma\left(x_{1}\right)=x_{2}$. Then $\sigma_{*}\left(y_{1}\right)=\sigma_{*}\left(f\left(x_{1}\right)\right)=f\left(\sigma\left(x_{1}\right)\right)=f\left(x_{2}\right)=y$. Therefore $Y$ is homogeneous. Because each $\sigma_{*}: Y \rightarrow Y$ is the restriction of a biholomorphic map $\sigma_{*}: G_{p}(V) \rightarrow G_{p}(V)$, the compact, $m$-dimensional irreducible analytic subset $Y$ of $G_{p}(V)$ is a connected smooth complex submanifold of $G_{p}(V)$. Because Aut $(X)$ operates transtiively on $X$ and $Y$ and commutes with $f: X \rightarrow Y$, the rank of the Jacobian matrix of $f$ is constant and equals the global rank of $f$ which is $m=\operatorname{dim} X=\operatorname{dim} Y$. Hence $f$ is locally biholomorphic. Because $f$ is also proper and because $Y$ is projective algebraic, the covering space $X$ is projective algebraic; Q.E.D.

Now the case of an ample holomorphic cotangent bundle will be considered. 
Lemma 6.3. Let $X$ and $Y$ be pure dimensional complex manifolds. Let $f: X \rightarrow Y$ be a smooth holomorphic map (i.e., a holomorphic immersion). If $T^{*}(Y)$ is ample, then $T^{*}(X)$ is ample.

Proof. Let $\omega_{0}, \cdots, \omega_{n}$ be finitely many holomorphic forms of bidegree $(1,0)$ on $Y$ such that $\omega_{0}(y), \cdots, \omega_{n}(y)$ generate $T_{y}^{*}(Y)$ over $\mathbf{C}$ for each $y \in Y$. Take $x \in X$. Take $a \in T_{x}^{*}(X)$. Define $y=f(x)$. Since $f$ is smooth, the induced linear map $\tilde{f}: \mid T_{y}^{*}(Y) \rightarrow T_{x}^{*}(X)$ is surjective. Hence $b \in T_{y}^{*}(Y)$ with $\tilde{f}(b)=a$ exists. Then $b=b_{0} \omega_{0}(y)+\cdots+b_{n} \omega_{n}(y)$ implies $a=f(b)=$ $b_{0} f *\left(\omega_{0}\right)(x)+\cdots+b_{n} f^{*}\left(\omega_{n}\right)(x)$. Hence $f^{*}\left(\omega_{0}\right)(x), \cdots, f^{*}\left(\omega_{n}\right)(x)$ generate $T_{x}^{*}(X)$ over $\mathbf{C}$ for each $x \in X$. The vector bundle $T^{*}(X)$ is ample; Q.E.D.

Let $X$ be a connected, compact, complex manifold of dimension $m$. Define

$$
\begin{aligned}
& V=V(X)=\Gamma\left(X, T^{*}(X)\right), \\
& q=q(X)=\operatorname{dim} V(X)<\infty .
\end{aligned}
$$

Then $q(X)$ is said to be the irregularity of $X$. If $X$ is Kaehlerian, then $2 q(X)$ is the first Betti number of $X$. Now, assume that $X$ is Kaehlerian. Then the Albanese Torus and the Jacobi map will be defined by the method of Weil [22|. The dual vector space $V^{*}$ will be called the Albanese vector space. Let $H_{1}(X, \mathbf{Z}), H_{1}(X, \mathbf{R})$ and $H_{1}(X, \mathbf{C})$ be the first (singular) homology groups of $X$ with coefficients in $\mathbf{Z}, \mathbf{R}$, and $\mathbf{C}$ respectively. The inclusions $\mathbf{Z} \subseteq \mathbf{R} \subseteq \mathbf{C}$ define homomorphisms

$$
H_{1}(X, \mathbf{Z}) \stackrel{\text { "⿱⺈ }}{\rightarrow} H_{1}(X, \mathbf{R}) \stackrel{12}{\rightarrow} H_{1}(X, \mathbf{C}) .
$$

Here $I_{2}$ is an $\mathbf{R}$-linear monomorphism. The rank of $H_{1}(X, \mathbf{Z})$ is $2 q$ and the dimensions of $H_{1}(X, \mathbf{R})$ over $\mathbf{R}$ and $H_{1}(X, \mathbf{C})$ over $\mathbf{C}$ are $2 q$. An $\mathbf{R}$-linear homomorphism $\alpha: H_{1}(X, \mathbf{R}) \rightarrow V^{*}$ is defined as follows. Take $c \in H_{1}(X, \mathbf{R})$. Represent $c$ as a differentiable cocycle $\gamma$ with coefficients in $\mathbf{R}$. Take any $\omega \in V$. Then

$$
\alpha(c)(\omega)=\int_{\gamma} \omega \in \mathbf{C}
$$

is well-defined, independent of the choice of $\gamma$. Hence $\alpha(c): V \rightarrow \mathbf{C}$ is $\mathbf{C}$-linear. Therefore $\alpha: H_{1}(X, \mathbf{R}) \rightarrow V^{*}$ is defined and obviously $\mathbf{R}$-linear. If $\alpha(c)=0$, then $\int_{y} \omega=0$ and $\int_{\gamma} \bar{\omega}=0$ for all $\omega \in V$. By de Rham, $\gamma \sim 0$ over $\mathbf{C}$, hence $t_{2}(c)=0$. Because $t_{2}$ is injective $c=0$. Hence $\alpha$ is injective. Consideration of dimensions shows

$$
\alpha: H_{1}(X, \mathbf{R}) \rightarrow V^{*}
$$


is an $\mathbf{R}$-linear isomorphism. Now $\iota_{1}\left(H_{1}(X, \mathbf{Z})\right)$ is a lattice in $H_{1}(X, \mathbf{R})$ (i.e., a discrete additive subgroup which generates $H_{1}(X, \mathbf{R})$ over $\left.\mathrm{R}\right)$. Hence

$$
\Delta=\alpha \circ \iota_{1}\left(H_{1}(X, \mathbf{Z})\right)
$$

is a lattice in $V^{*}$. The complex torus

$$
A(X)=V^{*} / \Delta
$$

is called the Albanese variety associated to $X$. Let $\rho: V^{*} \rightarrow A(X)$ be the residual map.

Now, a holomorphic map $J: X \rightarrow A(X)$ called the Jacobi map will be defined. Let $\psi: \hat{X} \rightarrow X$ be the universal covering of $X$. Take $a \in X$ and $\hat{a} \in \hat{X}$ with $\psi(\hat{a})=a$. At first, a holomorphic map $\hat{J}: \hat{X} \rightarrow V^{*}$ will be defined. Take $x \in \hat{X}$. Then $\hat{J}(x): V \rightarrow \mathbf{C}$ has to be defined as a $\mathbf{C}$-linear map. Take $\omega \in V$. Then $d \psi^{*}(\omega)=\psi *(d \omega)=0$. Hence, one and only one holomorphic function $f: \hat{X} \rightarrow \mathrm{C}$ exists such that $f(\hat{a})=0$ and $d f=\psi *(\omega)$. Define $\hat{J}(x)(\omega)=f(x)$. Obviously, $\hat{J}(x)$ is C-linear. Hence $\hat{J}: \hat{X} \rightarrow V^{*}$ is defined. Let $\omega_{1}, \cdots, \omega_{q}$ be a base of $V$. Let $\omega_{1}^{*}, \cdots, \omega_{q}^{*}$ be the dual base of $V^{*}$. Then holomorphic functions $f_{v}: X \rightarrow \mathrm{C}$ exist such that $d f_{v}^{\prime}=\psi^{*}\left(\omega_{v}\right)$ and such that $f_{v}(\hat{a})=0$. Hence

$$
\hat{J}(x)=\sum_{v=1}^{q} J(x)\left(\omega_{v}\right) \omega_{v}^{*}=\sum_{v=1}^{q} f_{v}(x) \omega_{v}^{*}
$$

for all $x \in \hat{X}$. Therefore $\hat{J}$ is holomorphic. Observe $\hat{J}(\hat{a})=0$.

Let $G$ be the group of all biholomorphic maps $g: \hat{X} \rightarrow \hat{X}$ with $\psi \circ g=\psi$. A map $\beta: G \rightarrow V^{*}$ is defined by $\beta(g)=J(g(\hat{a}))$ for $g \in G$. Obviously, $\beta(\mathrm{Id})=0$. Take $g \in G$ and $h \in G$. Take $\omega \in V$. Then $\psi^{*}(\omega)=d f$ with $f(\hat{a})=0$. Then

$$
d(f \circ h)=h^{*}(d f)=h^{*} \pi^{*}(\omega)=(\pi \circ h)^{*}(\omega)=\pi^{*}(\omega)=d f .
$$

Hence $f=f \circ h-f(h(a))$ which implies

$$
\begin{aligned}
\beta(h \circ g)(\omega) & =\hat{J}(h(g(\hat{a}))(\omega)=f(h(g(a)))=f(g(\hat{a}))+f(h(\hat{a})) \\
& =\hat{J}(g(\hat{a}))(\omega)+(h(\hat{a}))(\omega)=(\beta(g)+\beta(h))(\omega) .
\end{aligned}
$$

Hence, $\beta(h \circ g)=\beta(g)+\beta(h)$. Therefore $\beta: G \rightarrow V^{*}$ is a homomorphism. Let $\pi_{1}(X, a)$ be the fundamental group of $X$ at $a$. An isomorphism $\delta: \pi_{1}(X, a) \rightarrow G$ is defined as follows. Take $v \in \pi_{1}(X, a)$. Represent $v$ by a curve $\phi$ from $a$ to $a$, which lifts to a curve $\hat{\phi}$ from $\hat{a}$ to $x$ with $\psi \circ \hat{\phi}=\phi$. Then $\delta(v) \in G$ is uniquely defined by $x=\delta(v)(\hat{a})$. A homomorphism 
$\beta \circ \tilde{\delta}: \pi_{1}(X, a) \rightarrow V^{*}$ is defined. Let $C$ be the commutator subgroup of $\pi_{1}(X, a)$. Then an epimorphism $\varepsilon: \pi_{1}(X, a) \rightarrow H_{1}(X, \mathbf{Z})$ is defined with kernel $C$. Obviously, the kernel of $\beta \circ \delta$ contains $C$. Hence, a homomorphism

$$
\eta: H_{1}(X, \mathbf{Z}) \rightarrow V^{*}
$$

is defined such that $\eta \circ \varepsilon=\beta \circ \delta$. Now, $\eta=\alpha \circ \iota$ is claimed. Take $c \in H_{1}(X, \mathbf{Z})$. Then $c=\varepsilon(v)$, where $v \in \pi_{1}(X, a)$. Represent $v$ by a closed, differentiable curve $\phi$ from $a$ to $a$. Then $\phi$ can be considered to be a singular 1-simplex. As such it represents $c$ in $H_{1}(X, \mathbf{Z})$ and $\iota_{1}(c)$ in $H_{1}(X, \mathbf{R})$. Hence

$$
\left(\alpha \circ \iota_{1}(c)\right)(\omega)=\int_{\phi} \omega \quad \text { if } \omega \in V .
$$

As a curve, $\phi$ lifts to a curve $\hat{\phi}$ from $\hat{a}$ to $x$ in $\hat{X}$ such that $\psi \circ \hat{\phi}=\phi$. If $\omega \in V$, then $\psi^{*}(\omega)=d f$ with $f(\hat{a})=0$. Hence

$$
\int_{\phi} \omega=\int_{\hat{\phi}} \psi^{*}(\omega)=\int_{\phi} d f=f(x) .
$$

Now, $x=\delta(v)(\hat{a})$. Therefore

$$
\begin{aligned}
\eta(c)(\omega) & =\beta \circ \delta(v)(\omega)=\hat{J}(\delta(v)(\hat{a}))(\omega)=\hat{J}(x)(\omega) \\
& =f^{\prime}(x)=\int_{\phi} \omega=\left(\left(\alpha \circ \iota_{1}\right)(c)\right)(\omega) .
\end{aligned}
$$

Hence $\eta(c)=\alpha \circ \iota_{1}(c)$ and $\eta=\alpha \circ \iota_{1}$. Therefore

$$
\begin{aligned}
\Delta & =\alpha \circ \iota_{1}\left(H_{1}(X, \mathbf{Z})\right)=\eta\left(H_{1}(X, \mathbf{Z})\right)=\beta \circ \delta\left(\pi_{1}(X, a)\right) \\
& =\beta(G)=\{\hat{J}(g(a)) \mid g \in G\}=\hat{J}\left(\psi^{-1}(a)\right)
\end{aligned}
$$

because $G$ operates transitively on the fibers of $\psi$. Take $g \in G$. Take $\omega \in V$. Then $\psi^{*}(\omega)+d f$ with $f(\hat{a})=0$. Then $f=f \circ g-f(g(\hat{a}))$. Take $x \in \hat{X}$.

$$
\begin{aligned}
\hat{J}(g(x))(\omega) & =f(g(x))=f(x)+f(g(\hat{a})) \\
& =\hat{J}(x)(\omega)+\hat{J}(g(a))(\omega) \\
& =(\hat{J}(x)+\beta(g))(\omega) .
\end{aligned}
$$

Hence $\hat{J} \circ g-\hat{J}=\beta(g) \in \Delta$ is constant, which implies $\rho \circ \hat{J} \circ g=\rho \circ \hat{J}$. Because $G$ oparates transitively on all the fibers of $\psi$, one and only one map $J: X \rightarrow A(X)$, called the Jacobi map, exists such that $\hat{J} \circ \psi=\rho \circ \hat{J}$. 
Because $\psi$ is locally biholomorphic, $J$ is holomorphic. The following commutative diagram is established:

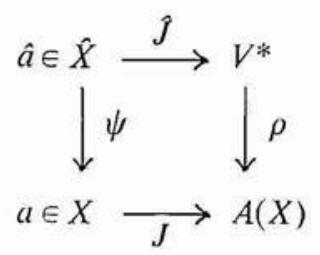

Both $\psi$ and $\rho$ are locally biholomorphic covering maps. $V^{*}$ and $A(X)$ are complex Lie groups under addition and $\rho$ is a holomorphic homomorphism with $0=\rho(0)=J(a)$. Because $\rho \circ \hat{J}$ does not depend on the choice of $\hat{a}$ in $\psi^{-1}(a)$, the Jacobi map $J$ depends on the choice of $a \in X$ only.

Now consider the case where $X$ is a complex torus. Then $\hat{X}$ is a complex vector space and $\psi$ is an additive homomorphism with kernel $\Delta_{0}=\psi^{-1}(0)$. If $t \in \hat{X}$, define the translation $g_{t}: \hat{X} \rightarrow \hat{X}$ by $g_{t}(x)=x+t$. Then $G=\left\{g_{t} \mid t \in \Delta_{0}\right\}$ is the group of covering transformations of $\psi$. Take $a=0=\psi(0) \in X$ and $\hat{a}=0 \in \hat{X}$. Take $\omega \in V$. Then $\psi^{*}(\omega)=d f$ with $f: \hat{X} \rightarrow C$ holomorphic and with $f(0)=0$. Since $f=f \circ g_{t}-f(t)$ for all $t \in \Delta_{0}$, and since $\Delta_{0}$ is a lattice in $\hat{X}$, the holomorphic function $f$ is linear. A map $\lambda: V \rightarrow X^{*}$ is defined by $\lambda(\omega)=f$. Obviously, $\lambda$ is linear. If $\lambda(\omega)=0$, then $\psi^{*}(\omega)=d \lambda(\omega)=0$. Hence $\omega=0$. So $\lambda$ is injective. If $f \in X^{*}$, then $f=f \circ g_{t}-f(t)$ for all $t \in \hat{X}$. Hence, $\omega \in V$ exists such that $\psi^{*}(\omega)=d f$. Therefore, $f=\lambda(\omega)$. The map $\lambda$ is an isomorphism. The dual map $\lambda^{*}: X \rightarrow V^{*}$ is also isomorphic. Take $x \in \hat{X}$ and $\omega \in V^{*}$. Then

$$
\lambda^{*}(x)(\omega)=\lambda(\omega)(x)=\hat{J}(x)(\omega)
$$

Therefore, $\hat{J}=\lambda^{*}: \hat{X} \rightarrow V^{*}$ is a linear isomorphism. The kernel $\Delta_{0}$ of $\psi$ is mapped onto the kernel $\Delta=\hat{J}\left(\psi^{-1}(0)\right)=\hat{J}\left(\Delta_{0}\right)$ of $\rho$. Therefore $J: X \rightarrow A(X)$ is a Lie group isomorphism. In this sense, a complex torus is its own Albanese variety.

Proposition 6.4. Let $X$ be a connected, compact Kaehler manifold. Then the holomorphic cotangent bundle $T^{*}(x)$ is ample if and only if $X$ can be holomorphically immersed into a complex torus.

Proof. The cotangent bundle of a complex torus is ample. Hence, if $X$ is holomorphically immersed into a complex torus, then $T^{*}(X)$ is ample by Lemma 6.3. Assume that $T^{*}(X)$ is ample. Consider diagram (6.3). It will be shown that $J$ is smooth. Because $\psi$ and $\rho$ are locally biholomorphic, 
it suffices to show that $\hat{J}$ is smooth. Take $\hat{x} \in \hat{X}$ and define $x=\psi(\hat{x})$. Take a base $\omega_{1}, \cdots, \omega_{q}$ of $V$ such that $\omega_{1}(x), \cdots, \omega_{m}(x)$ is a base of $T_{x}^{*}(X)$. Because $\psi$ is locally biholomorphic, $\psi^{*}\left(\omega_{1}\right)(\hat{x}), \cdots, \psi^{*}\left(\omega_{m}\right)(\hat{x})$ is a base of $T_{\hat{x}}^{*}(\hat{X})$. Now, $\psi^{*}\left(\omega_{\mu}\right)=d f_{\mu}$ with $f_{\mu}(\hat{a})=0$ for $\mu=1, \cdots, q$. Let $\omega_{1}^{*}, \cdots, \omega_{q}^{*}$ be the dual base to $\omega_{1}, \cdots, \omega_{q}$. Then $\hat{J}$ is given by (6.2). Because

$$
\left(d f_{1}\right)(\hat{x}) \wedge \cdots \wedge\left(d f_{m}\right)(\hat{x})=\psi^{*}\left(\omega_{1}\right)(\hat{X}) \wedge \cdots \wedge \psi^{*}\left(\omega_{m}\right)(\hat{x}) \neq 0
$$

the Jacobian matrix of $\hat{J}$ has at least rank $m$ (hence rank $m$ ) at $\hat{x}$. Therefore $\hat{J}$ is smooth; Q.E.D.

Theorem 6.5. Let $X$ be a compact, connected, complex manifold with an ample holomorphic cotangent bundle. Take $\kappa=\left(\kappa_{1}, \cdots, \kappa_{q}\right)$ with $0 \leqq \kappa_{\mu} \in \mathbf{Z}$ and with $\sigma(\kappa)=m=\operatorname{dim} X$. Then

$$
(-1)^{m} C_{\kappa}(X) \geqq 0 .
$$

Moreover, if $X$ is Kaehlerian and if at least one Chern number $C_{\kappa}(X) \neq 0$ is not zero, then $X$ is projective algebraic.

Remark. Bochner proved $(-1)^{m} \chi(X) \geqq 0$ in [3].

Proof. Because $T^{*}(X)$ is ample, Proposition 4.2 implies

$$
(-1)^{m} \dot{C}_{\kappa}(X)=C_{\kappa}\left(T^{*}(X)\right) \geqq 0 .
$$

The remaining statement follows from the Main Theorem, Q.E.D.

Let $X$ be a connected, compact Kaehler manifold of dimension $m$ with ample cotangent bundle. Then $m \leqq q(X)$. If $q(X)=m$, then $X$ is a complex torus, because in this case the Jacobi map is biholomorphic. Now the case $q(X)=m+1$ will be studied. At first a preparation:

Lemma 6.6. Let $X$ be a complex space. Let

$$
0 \rightarrow L \rightarrow X \times V \stackrel{e}{\rightarrow} E \rightarrow 0
$$

be an exact sequence of holomorphic vector bundles where $E$ and $L$ have fiber dimensions $p$ and 1 respectively and where Vhas dimension $n=p+1$. The exterior product on forms induces an exterior product on the direct sum of the refined de Rham groups. Then $\hat{c}_{v}(E)=\hat{c}_{1}(E)^{v}$ for $v=1, \cdots, p$. If $\kappa=\left(\kappa_{1}, \cdots, \kappa_{q}\right)$ with $0 \geqq \kappa_{v} \in \mathbf{Z}$, then

$$
\hat{c}_{\kappa}(E)=\hat{c}_{1}(E)^{\sigma(\kappa)} \text {. }
$$

Proof. By Bott and Chern [5], Proposition 1.5

$$
\hat{c}(E) \wedge \hat{c}(L)=\hat{c}(X \times V)=1 .
$$


Hence

$$
\hat{c}_{v}(E)+\hat{c}_{v-1}(E) \wedge \hat{c}_{1}(L)=0 \text { for } v>0 .
$$

Especially, $\hat{c}_{1}(E)=-\hat{c}_{1}(L)$ which implies

$$
\hat{c}_{v}(E)=\hat{c}_{v-1}(E) \wedge \hat{c}_{1}(E) .
$$

By induction

$$
\hat{c}_{v}(E)=\hat{c}_{1}(E)^{v} \quad \text { for } \quad v=1, \cdots, p .
$$

Because $\sigma(\kappa)=\sum_{v=1}^{p} v \kappa_{v}$, this implies

$$
\hat{c}_{\kappa}(E)=\prod_{\nu=1}^{p} \hat{c}_{\kappa}(E)^{\kappa(v)}=\hat{c}_{1}(E)^{\sigma(\kappa)} ; \text { Q.E.D. }
$$

Proposition 6.7. Let $X$ be an m-dimensional, connected, compact, complex manifold with an ample holomorphic cotangent bundle and with irregularity $q(X)=m+1$. Let $\chi(X)$ be the Euler characteristic of $X$. Take any $\kappa=\left(\kappa_{1}, \cdots, \kappa_{m}\right)$ with $0 \leqq \kappa_{\mu} \in \mathbf{Z}$ and $\sigma(\kappa)=m$. Then

$$
C_{\kappa}(X)=\chi(X) \text {. }
$$

Proof. Define $V=\Gamma\left(X, T^{*}(X)\right)$. An amplification

$$
0 \rightarrow S \rightarrow X \times V \stackrel{\mathfrak{c}}{\rightarrow} T^{*}(X) \rightarrow 0
$$

is given, where $e$ is the evaluation map. Because $\operatorname{dim} V=q(X)=m+1$ and because $T^{*}(X)$ has fiber dimension $m$, the bundle $S$ is a line bundle. Hence $\hat{c}_{\kappa}\left(T^{*}(X)\right)=\hat{c}_{1}\left(T^{*}(X)\right)^{m}$ for all $\kappa$ with $\sigma(\kappa)=m$. Because $\hat{c}_{\kappa}(X)$ $=(-1)^{m} \hat{c}_{\kappa}\left(T^{*}(X)\right)$ all the Chern class of $T(X)$ are the same. Hence all the Chern numbers of $X$ are the same, which implies

$$
C_{\kappa}(X)=\int_{X} c_{m}(X)=\chi(X) ; \text { Q.E.D. }
$$

If the holomorphic cotangent bundle of a connected, compact Kaehler manifold $X$ of dimension $m$ is ample and if $q(X)=m+1$, then $X$ is immersed into the Albanese variety $A(X)$, which is a complex torus of dimension $m+1$. Moreover, if $\chi(X) \neq 0$, then $X$ is projective algebraic, and, consequently, $A(X)$ is projective algebraic (Weil [21]). Now it will be shown that every projective algebraic torus of dimension $\geqq 3$ can be almost realized this way:

Theorem 6.8. Let $B$ be an abelian variety of dimension $m+1>2$. Then a connected, compact, m-dimensional, projective algebraic manifold 
$X$ with $q(X)=m+1$ and $\chi(X) \neq 0$ exists such that the holomorphic cotangent bundle of $X$ is ample. Moreover, a finite subgroup $D$ of the Albanese variety $A(X)$ of $X$ exists such that $B=A(X) / D$.

Proof. Because $B$ is projective algebraic, it can be considered to be a smooth, compact, complex submanifold of complex projective space. According to the theorem of Bertini, a hyperplane section $Y$ exists in $B$ such that $Y$ is a compact, connected, $m$-dimensional, smooth, complex submanifold of $B$. Recall that the torus $B$ is a complex Lie group under addition. Take a point $a \in Y$. Define a biholomorphic map $\mu: B \rightarrow B$ by $\mu(x)=x-a$ for $x \in B$. Then $X=\mu(Y)$ is a compact, connected, $m$-dimensional, smooth, complex submanifold of $B$. Since $B$ is projective algebraic, $X$ is projective algebraic. The holomorphic cotangent bundle $T^{*}(X)$ of $X$ is ample by Proposition 6.4. Observe that $0=\mu(a) \in X$. According to the Lefschetz theorem (Milnor [12]), the vth Betti numbers for $v=0,1, \cdots, m-1$ are

$$
\begin{aligned}
& b_{v}(X)=b_{v}(Y)=b_{v}(B)=\left(\begin{array}{c}
2 m+2 \\
v
\end{array}\right), \\
& b_{m}(X)=b_{m}(Y) \geqq b_{m}(B)=\left(\begin{array}{c}
2 m+2 \\
m
\end{array}\right) .
\end{aligned}
$$

Since $m>1$ by assumption, $b_{1}(X)=b_{1}(B)=2(m+1)$. Hence $q(X)=\frac{1}{2} b_{1}(X)=m+1$. Poincaré duality implies

$$
b_{2 m-v}(X)=b_{v}(X)=\left(\begin{array}{c}
2 m+2 \\
v
\end{array}\right)=\left(\begin{array}{c}
2 m+2 \\
2 m+2-v
\end{array}\right)
$$

for $y=0,1, \cdots, m-1$. Therefore

$$
\begin{aligned}
\chi(X) & =\sum_{v=0}^{2 m}(-1)^{v} b_{v}(X) \\
& =\sum_{v=0}^{m-1}(-1)^{v}\left(\begin{array}{c}
2 m+2 \\
v
\end{array}\right)+(-1)^{m} b_{m}(X)+\sum_{v=0}^{m-1}(-1)^{v}\left(\begin{array}{c}
2 m+2 \\
2 m+2-v
\end{array}\right) \\
& =\sum_{v=0}^{2 m+2}(-1)^{v}\left(\begin{array}{c}
2 m+2 \\
v
\end{array}\right)+(-1)^{m}\left(b_{m}(X)-\left(\begin{array}{c}
2 m+2 \\
m
\end{array}\right)+\left(\begin{array}{c}
2 m+2 \\
m-1
\end{array}\right)\right. \\
& =(-1)^{m}\left(b_{m}(X)-b_{m}(B)+\frac{(2 m+2) !}{(m+1) !(m+2) !}\right) .
\end{aligned}
$$

Because $b_{m}(X) \geqq b_{m}(B)$, the characteristic $\chi(X)$ is not zero. 
Since $B$ is a complex torus, a complex vector space $W$ of dimension $m+1$ and a lattice $\Delta_{B}$ in $W$ exist such that $B=W / \Delta_{B}$. Let $\eta: W \rightarrow B$ be the residual map. Let $V(B)$ and $V(X)$ be the vector spaces of holomorphic forms of bidegree $(1,0)$ on $B$ and $X$ respectively. Let $j: X \rightarrow B$ be the inclusion map. Then $j^{*}: V(B) \rightarrow V(X)$ is a linear homomorphism. It is claimed that $j^{*}$ is an isomorphism.

Take $\omega \in V(B)$ with $j^{*}(\omega)=0$. Then $\eta^{*}(\omega)=d \beta$ with $\beta(0)=0$. The map $\beta: W \rightarrow \mathbf{C}$ is linear. Assume that $\beta \neq 0$. Because $0 \in X$, the inverse image $\eta^{-1}(X)$ has a component $L$ with $0 \in L$. Let $j_{L}: L \rightarrow W$ be the inclusion. $L$ is a smooth complex submanifold of dimension $m$ of $W$. The covering map $\eta: W \rightarrow B$ restricts to a covering map $\eta_{L}: L \rightarrow X$, with $j \circ \eta_{L}=\eta \circ j_{L}$. Therefore

$$
\begin{aligned}
d \beta \circ j_{L} & =j_{L}^{*} d \beta=j_{L}^{*} \circ \eta^{*}(\omega)=\left(\eta \circ j_{L}\right)^{*}(\omega) \\
& =\left(j \circ \eta_{L}\right)^{*}(\omega)=\eta_{L}^{*}\left(j_{L}^{*}(\omega)\right)=\eta_{L}^{*}(0)=0 .
\end{aligned}
$$

Hence $\beta \circ j_{L}$ is constant. Because $\beta \circ j_{L}(0)=\beta(0)=0$, the function $\beta \circ j_{L} \equiv 0$ vanishes. Hence $L \subseteq \operatorname{ker} \beta$. Because $\operatorname{ker} \beta$ is a linear subspace of dimension $m$, and $\operatorname{dim} L=m$, this implies $L=\operatorname{ker} \beta$. The linear subspace $L$ is a submodule of $W$. Hence $X=\eta(L)$ is a subgroup of $B$. The sequence

$$
0 \rightarrow \Delta_{B} \cap L \rightarrow L \stackrel{\eta_{L}}{\rightarrow} X \rightarrow 0
$$

is exact. Therefore $X$ is a complex torus. Since $\chi(X) \neq 0$, this is impossible. Hence $\beta \equiv 0$, which implies $\eta^{*}(\omega) \equiv 0$ and $\omega \equiv 0$. The map $j^{*}$ is injective. Because $V(B)$ and $V(X)$ have dimension $m+1$

$$
j^{*}: V(B) \rightarrow V(X)
$$

is an isomorphism. Define the dual isomorphism as

$$
j_{*}=\left(j^{*}\right)^{*}: V(X)^{*} \rightarrow V(B)^{*} .
$$

The inclusion map $j: X \rightarrow B$ induces homomorphisms $j_{*}$ on the homology groups. The following diagram is established.

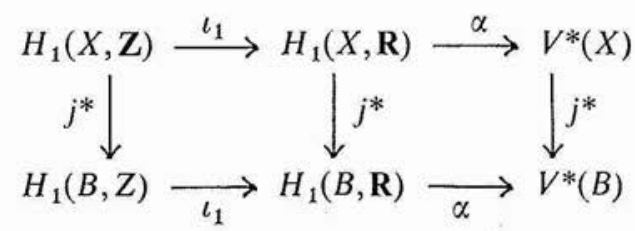


with $\iota_{1} \circ j_{*}=j_{*} \circ \iota_{1}$. Take $c \in H_{1}(X, \mathbf{R})$. Represent $c$ by a differentiable cocycle with coefficients in $R$. Take $\omega \in V(B)$. Then

$$
j_{*}(\alpha(c))(\omega)=\alpha(c)\left(j^{*}(\omega)\right)=\int_{\gamma} j^{*}(\omega)=\int_{j_{*} \gamma} \omega=\alpha\left(j_{*}(c)(\omega)\right.
$$

Therefore $j_{*} \circ \alpha=\alpha \circ j_{*}$. The diagram (6.5) commutes. Let $\Delta(X)$ and $\Delta(B)$ be the Albanese lattices of $X$ and $B$ respectively. Then

$$
\begin{aligned}
j_{*}(\Delta(X)) & =j_{x} \circ \alpha \circ \iota_{1}\left(H_{1}(X, \mathbf{Z})\right)=\alpha \circ \iota_{1} \circ j_{*}\left(H_{1}(X, \mathbf{Z})\right) \\
& \subseteq \alpha \circ \iota_{1}\left(H_{1}(B, \mathbf{Z})\right)=\Delta(B)
\end{aligned}
$$

A homomorphism of exact sequences is defined

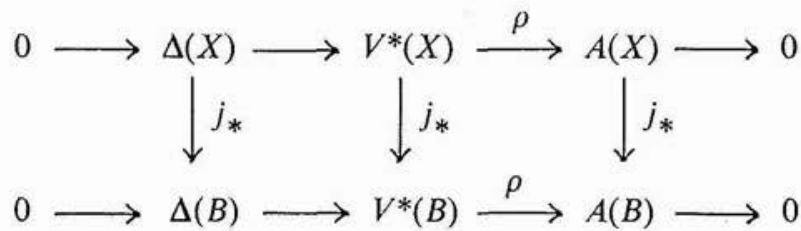

Because $j_{*}: V^{*}(X) \rightarrow V^{*}(B)$ is an isomorphism, $j_{*}: A(X) \rightarrow A(B)$ is surjective. Because $A(X)$ and $A(B)$ have the same dimension $m+1$, and because all fibers of $j_{*}$ have the same number of points, the surjective holomorphic map $j_{*}$ is light. Hence the kernel $D$ of $j_{*}$ is finite. Because $B$ is a complex torus the Jacobi map $J_{B}: B \rightarrow A(B)$ defined at $0 \in B$ is a Lie group isomorphism. Define $\lambda=J_{B}^{-1} \circ j_{*}$. An exact sequence

$$
0 \rightarrow D \rightarrow A(X) \stackrel{\lambda}{\rightarrow} B \rightarrow 0
$$

is defined, where $\lambda$ is a Lie group homomorphism, Q.E.D.

\section{NOTES}

1. Only reduced complex spaces with a countable base of open sets are admitted in this paper.

2. A holomorphic map $f: X \rightarrow Y$ is said to be regular, if its Jacobian matrix has constant rank $n$ with $n=\operatorname{dim} Y$.

3. If $\psi$ is a form, define $\psi^{t}=\psi \wedge \cdots \wedge \psi(t$-times $)$.

\section{REFERENCES}

[1] AndReotti, A., and W. Stoll, "Analytic and algebraic dependence of meromorphic functions," Lecture Notes in Mathematics 234 (Berlin-Heidelberg-New. York: Springer-Verlag, 1971), 390 pages. 
[2] Bloom, T., and M. Herrera, "DeRham cohomology of an analytic space," Invent. Math. 7 (1969), 275-296.

[3] Bochner, S., "Euler-Poincaré characteristic for locally homogeneous and complex spaces," Ann. of Math. 51 (1950), 241-261.

[4] Borel, A., and R. Remmert, "Ueber kompakte, homogene Kählersche Mannigfaltigkeiten," Math. Annalen 145 (1962), 429-439.

[5] BotT, R., and S. S. CHERN, "Hermitian vector bundles and the equidistribution of the zeroes of their holomorphic sections," Acta Math. 114 (1965), 71-112.

[6] Cowen, M., "Value distribution," M.I.T. Thesis (1971), p. 90.

[7] Goto, M., "On algebraic homogeneous spaces," Am. J. Math. 76 (1954), 811-818.

[8] Grauert, H., and R. Remmert, "Úber kompakte homogene komplexe Mannigfaltigkeiten," Archi. Math. 13 (1962), 482-507.

[9] Herrera, M., "Integration on a semi-analytic set," Bull. Soc. Math. France 94 (1966), 141-180.

[10] KING, J., "The currents defined by analytic varieties," Acta Math. 127 (1971), 185-220.

[11] Lelong, P., "Intégration sur une ensemble analytique complexe", Bull. Soc. Math. France 85 (1957), 328-370.

[12] Milnor, J., "Morse theory," Ann. of Math. Studies 51 (Princeton University Press, 1963), 160 pages.

[13] MolšEzon, B., "On $n$-dimensional, compact complex manifolds having $n$ algebraically independent meromorphic functions," Isv. Nauk SSSR. Ser. Mat. 30 (1966), 133-174, 345-386, 621-656.

[14] Remmert, R., "Holomorphe und meromorph Abbildungen komplexer Räume,” Math. Annalen 133 (1957), 328-370.

[15] Stoll, W., "A general first main theorem of value distribution," Acta Math. 118 (1967), 111-191.

[16] —- "About value distribution of holomorphic maps into the projective space," Acta Math. 123 (1969), 83-114.

[17] —-, "Value distribution of holomorphic maps into compact complex manifolds," Lecture Notes in Mathematics 135 (BerlinHeidelberg-New York: Springer-Verlag, 1970), 267 pages.

[18] — - "Value distribution of holomorphic maps,"' Several complex Variables I, Maryland 1970, Lecture Notes in Mathematics 155 (Berlin-Heidelberg-New York: Springer-Verlag, 1970), 165-170.

[19] — "Fiber integration and some of its applications," Symposium on Several Complex Variables, Park City Utah 1970, Lecture 
Notes in Mathematics 184 (Berlin-Heidelberg-New York: Springe1-Verlag, 1971), 109-120.

[20] TunG, CH., "The first main theorem on complex spaces," Notre Dame Thesis, 1973.

[21] WeIL, A., "Introduction à l'étude des variétés kähleriennes," Publ. Inst. Math. Univ. de Nangaco IV. (Paris: Hermann, 1958).

[22] —_, "On Picard Varieties," Amer. J. Math. 74 (1952), 865-894.

University of Notre DAME 